\title{
GEOSECS PACIFIC RADIOCARBON
}

\author{
H G ÖSTLUND
}

Tritium Laboratory

Rosenstiel School of Marine and Atmospheric Science

University of Miami, Miami, Florida 33149

and

\section{MINZE STUIVER}

Quaternary Isotope Laboratory

University of Washington, Seattle, Washington 98195

In the preceding paper (Stuiver and Östlund, this issue), some of the general features of the Geochemical Ocean Section Study (GEOSECS) were outlined and results were listed for the Atlantic Ocean. This paper will deal with the second major cruise, which was described by Craig and Turekian (1976), covering the Pacific Ocean in 1973-74 (cf fig 1).

The sampling and measurement techniques were not substantially changed from what was described in the Stuiver and östlund paper (1980). This expedition resulted in a larger number of ${ }^{14} \mathrm{C}$ measurements than the Atlantic due to the larger size of the Pacific Ocean. The areas visited were selected for ocean-wide coverage, allowing coherent sections to be drawn. Somewhat more intensified sampling was performed in particularly interesting areas, eg, across the Equatorial Current system, and on the Antarctic leg with some emphasis on the Antarctic Circumpolar Current.

A large number of Pacific radiocarbon measurements have been made during the past 20 years at Scripps Institution of Oceanography of the University of California by $\mathrm{H} \mathrm{E}$ Suess and his co-workers; a comprehensive summary was released by Linick (1978). Other Pacific data are reported by Rafter and O'Brien $(1970 ; 1972)$, and by Fairhall, Young, and Bradford (1972) who measured a sizeable number of samples collected on ships of opportunity. In contrast to previous studies, the GEOSECS expedition was more of a quasi-synoptic study of the entire ocean, with a coverage dense enough and resolution good enough to describe the major water masses in some detail. The average ${ }^{14} \mathrm{C}$ levels of Pacific polar $\left(>50^{\circ} \mathrm{S}\right)$, gyre $\left(50^{\circ} \mathrm{S}-10^{\circ} \mathrm{S}\right.$ and $\left.10^{\circ} \mathrm{N}-50^{\circ} \mathrm{N}\right)$ and tropical waters $\left(10^{\circ} \mathrm{S}-10^{\circ} \mathrm{N}\right)$ are given separately (Stuiver, Östlund, and McConnaughy, 1980). Estimates of nuclear bomb ${ }^{14} \mathrm{C}$ inventories were made in this paper, and also by Broecker, Peng, and Engh (in press).

\section{THE $\Delta^{14} \mathrm{C}$ SCALE}

In this paper, we have, in principle, followed the recommendations by Stuiver and Robinson (1974) which means that the ${ }^{14} \mathrm{C}$ values are recalculated to a standard wood $\left(\delta^{13} \mathrm{C}=-25 \%\right)$, and they relate to activities at time of sampling - $1973-1974 . \delta^{13} \mathrm{C}$ values are not listed since they refer to each specific $\mathrm{CO}_{2}$ gas sample at time of counting. That value may differ slightly from the $\delta^{13} \mathrm{C}$ of the total $\mathrm{CO}_{2}$ in the original 


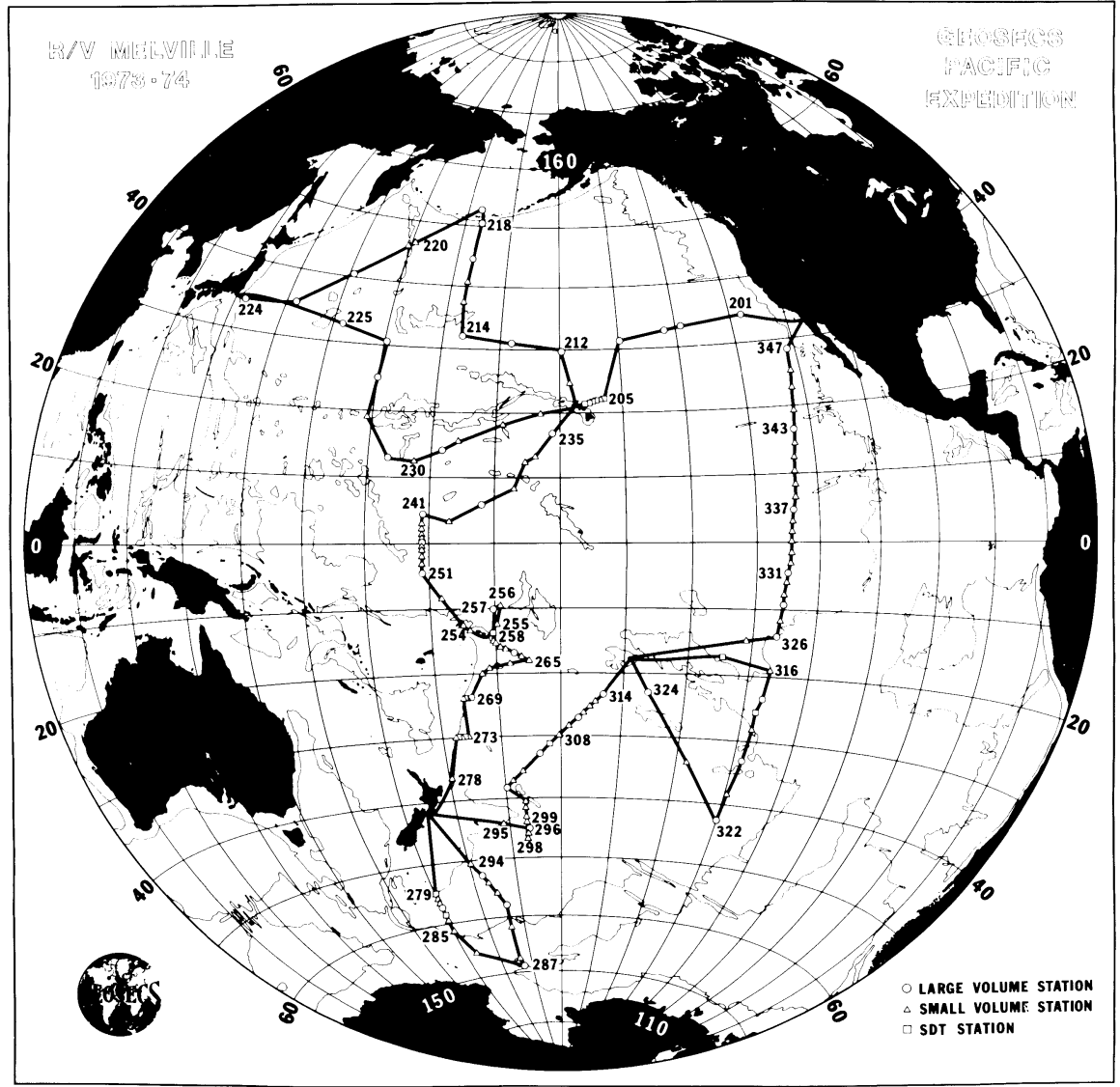

Fig 1. GEOSECS Pacific Track 1973-1974.

seawater sample, due to an albeit minor fractionation during the $\mathrm{CO}_{2}$ extraction.* Appropriate corrections for the decay of the NBS ${ }^{14} \mathrm{C}$ standard have also been made. The $\Delta^{14} \mathrm{C}$ scale is, for all practical purposes, the same as the one proposed by Broecker and Olson (1961).

\section{EXPLANATION OF THE TABLES}

All data on position, depths, and hydrography and total $\mathrm{CO}_{2}$ have been furnished by the GEOSECS Operations Group (now Physical and Chemical Ocean Data Facility) at Scripps Institution of Oceanography, which handled the logistics and operations on board the ship and also serves as a temporary repository for all GEOSECS data. The following explains the column headings:

Position: Given in degrees and minutes. The ship frequently drifted during station time, so the position is defined to no better than \pm a few minutes.

* Water samples for precision ${ }^{13} \mathrm{C}$ measurements were collected separately and are being measured by Peter Kroopnick (Univ Hawaii, pers commun). 
SMPL \#: This is the operational sample number, in which the two last digits indicate the Gerard barrel number and the preceding digits the case number. The first on station 201 is sample no. 1187: ie, cast \#11, Gerard \#87.

DEPTH M: Given in meters as calculated from density and pressure.

POT T DEG C: Potential temperature in degrees centigrade.

SAL $\%$ : Salinity in unit $\mathrm{g} / \mathrm{kg}$ seawater.

SIGMA THETA: Deviation from unity, in per mille, of the relative density in $\mathrm{g} / \mathrm{ml}$ where $\mathrm{ml}$ has the old value of $1.000027 \mathrm{~cm}^{3}$.

TCO2 $\mu \mathrm{M}$ : The total amount of inorganic carbon in $\mu$-moles per $\mathrm{kg}$ of seawater. All TCO2 data listed are still preliminary. At time of writing, a final re-evaluation is being made on all GEOSECS oceanic CO. measurements. This will most likely result in minor adjustments on these numbers.

DC14 $\%$ : This is $\Delta^{14} \mathrm{C}$ on the scale that was defined above. The accuracy is typically $\pm 4 \%$ and precision $\pm 3.5 \%$.

Ci14 IAB \#: This column lists MI, for the Miami Laboratory and QL for the Washington Laboratory with numbers referring to our laboratory journals.

\section{THE SECTIONS}

The track of the Pacific GEOSECS expedition allowed three vertical sections to be made, plates 1, 2, and 3. The horizontal scales are proportional to distances between stations along the track.

One of the striking features in the section Western Pacific (pl 1) is, for instance, that the northern part does not exhibit the deep convection and renewal of deep waters that is so prominent in the Atlantic Ocean (cf pl 1, Stuiver and Östlund, 1980). Furthermore, in the Pacific N-S sections (pls 1 and 2), it is seen that the oldest waters are at mid-depth and in the northern parts, while in the Atlantic the mid-depth tongue of North Atlantic Deep Water is younger than below and above. Previous attempts of using ${ }^{14} \mathrm{C}$ measurements to estimate the time scales of the Pacific deep circulation have been made by Craig $(1969 ; 1971)$ and Bien, Rakestraw, and Suess (1960, 1963a, b; 1965). A renewed effort to use the recent available data was made by Rooth, Fine, and Östlund (1980) wherein the potential density has been substituted for depth coordinate. This approach yields a more realistic estimate on the time scales of renewal and circulation of the bulk of the old Pacific deep waters. They find vertical convergence of the below-themocline waters in the North Pacific, balanced by southerly flow at mid-depth (cf also Reid, 1973).

\section{ACKNOWLEDGMENTS}

The work of the first author was supported by grants GX-28165, IDO71-04199, and OCE76-03050, from the International Decade of Ocean Exploration of the National Science Foundation. The work of the second author was supported by grants GX-28166, IDO71-04200, and OCE76-03930 from the same agency. Sample collection at sea was carried out by personnel of the GEOSECS Operations Group, with the late Arnold Bainbridge of the Scripps Institution of Oceanography directing the fieldwork. 
REFERENCFS

Bien, G S, Rakestraw, N W, and Suess, H E, 1960, Radiocarbon concentration in Pacific Ocean water: Tellus, v 12, p 436-443.

1963a, Radiocarbon dating of deep water of the Pacific and Indian oceans: Inst Occanog Bull, v 16, p 12-16. Also in Radioactive dating, Vicnna, IAEA, p $159-173$.

1963b, Natural radiocarbon in the Pacific and Indian oceans: Nuclear Sci ser rept no. 38, pub 1075, NAS-NRC, Washington, DC, p 152-160.

1965, Radiocarbon in the Pacific and Indian oceans and its relation to deep water movements, in International conf of radiocarbon and oceanog, 6th, Proc: v 1, suppl, p R25-R37.

Brocker, W S and Olson, I A, 1961, Lamont radiocarbon measurements VIII: Radiocarbon, v 3, p 176-204.

Broecker, W S, Peng, T H, and Engh, Richard, 1980, Modeling the carbon system, in Stuiver, Minze and Kra, Renee, eds, International radiocarbon conference, 10th, Proc: Radiocarbon, v 22, no. 3.

Craig, Harmon, 1969, Abyssal carbon and radiocarbon in the Pacific: Jour Geophys Research, v 74, p 4910-4920.

1971, Son of abyssal carbon: Jour Geophys Research, v 76, p 5133-5139.

Craig, Harmon and Turekian, K K, 1976, The GEOSECS Program: 1973-1978: Earth Planctary Sci Letters, v 32, p $217-219$.

Fairhall, A W, Young, A W, and Bradford, P A, 1972, Radiocarbon in the sea, in Rafter, 'I' $A$ and Grant-Taylor, $T$, eds, International conf on radiocarbon dating, 8th, Proc: Wellington, Royal Soc New Zealand, v 1, p C2-C16.

Linick, 'I W, 1978, La Jolla measurements of radiocarbon in the oceans: Radiocarbon, v 20, p 333-359.

Rafter, T A and O'Brien, B J, 1970, Exchange rates between the atmosphere and the ocean as shown by recent C-14 measurements in the South Pacific, in Olsson, I U, ed, Radiocarbon variations and absolute chronology, Nobel symposium, 12th, Proc: New York, John Wiley \& Sons, p 355-377.

$1972,{ }^{14} \mathrm{C}$ measurements in the South Pacific Occan - a recalculation of the

exchange rates between the atmosphere and the ocean, in Rafter, $\mathrm{T} A$ and GrantTaylor, T, eds, International conference on radiocarbon dating, 8th, Proc: Wellington, Royal Soc New Kealand, v 1, p C17-C42.

Reid, J L, 1973, Transpacific hydrographic sections at Lats $43^{\circ} \mathrm{S}$ and $28^{\circ} \mathrm{S}$ : the SCORPIO Expedition - III. Upper water and a note on southward flow at middepth: Decp-sea Research, v 20, p 39-49.

Rooth, C G H, Fine, R A, and Östlund, H G, in press, Abyssal mixing and circulation in the North Pacific from radiocarbon data: Jour Marine Research, in press.

Stuiver, Minze and Östlund, H G, 1980, GEOSECS Atlantic radiocarbon: Radiocarbon, v 22, no. 1 , p $1-24$

Stuiver, Minze, Östlund, H G, and McConnaughy, T A, 1980, GEOSECS Atlantic and Pacific ${ }^{14} \mathrm{C}$ distribution, in SCOPE Proc, La Jolla modeling conference, March 1979: New York, John Wiley \& Sons, in press.

Stuiver, Minze and Robinson, S W, 1974, University of Washington GEOSECS North Atlantic carbon-14 results: Earth Planctary Sci Letters, v 23, p 87-90. 


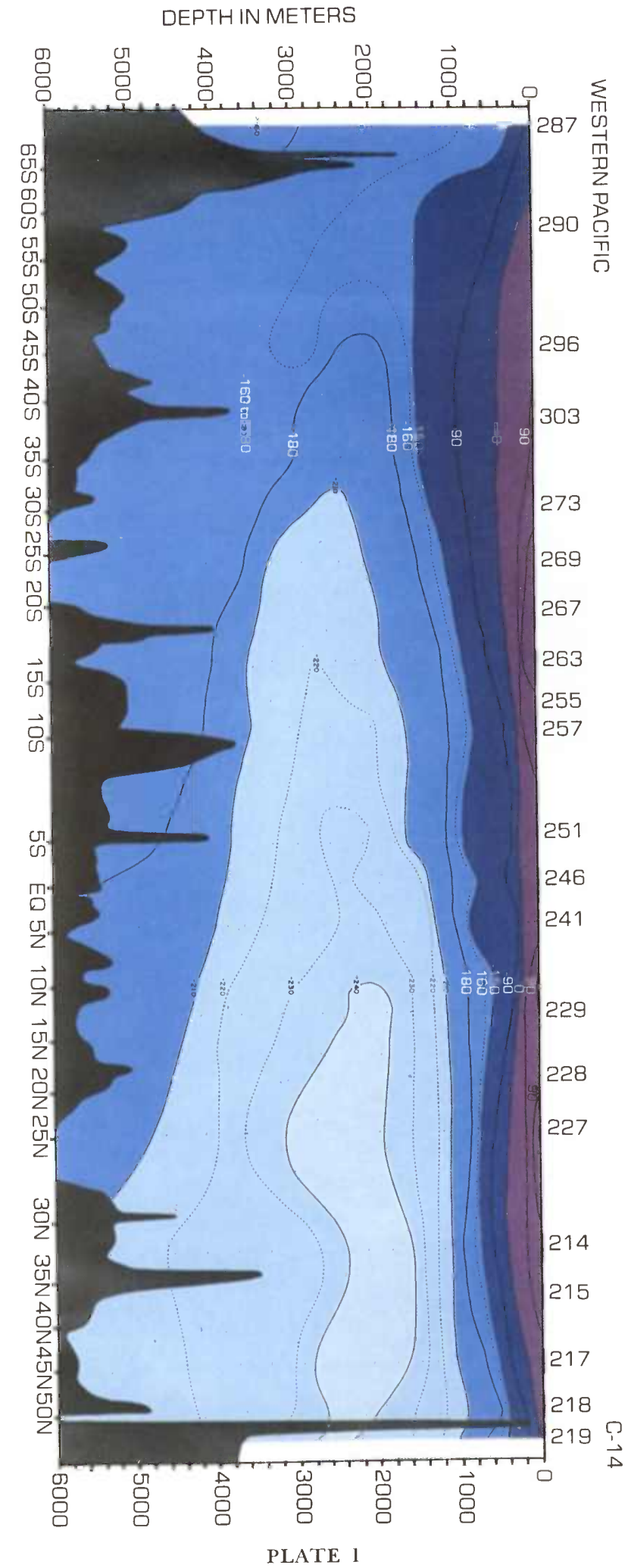

Western Pacific Ocean; N-S section. Isolines are in $\Delta^{14} \mathrm{C}$ units. 
DEPTH IN METERS

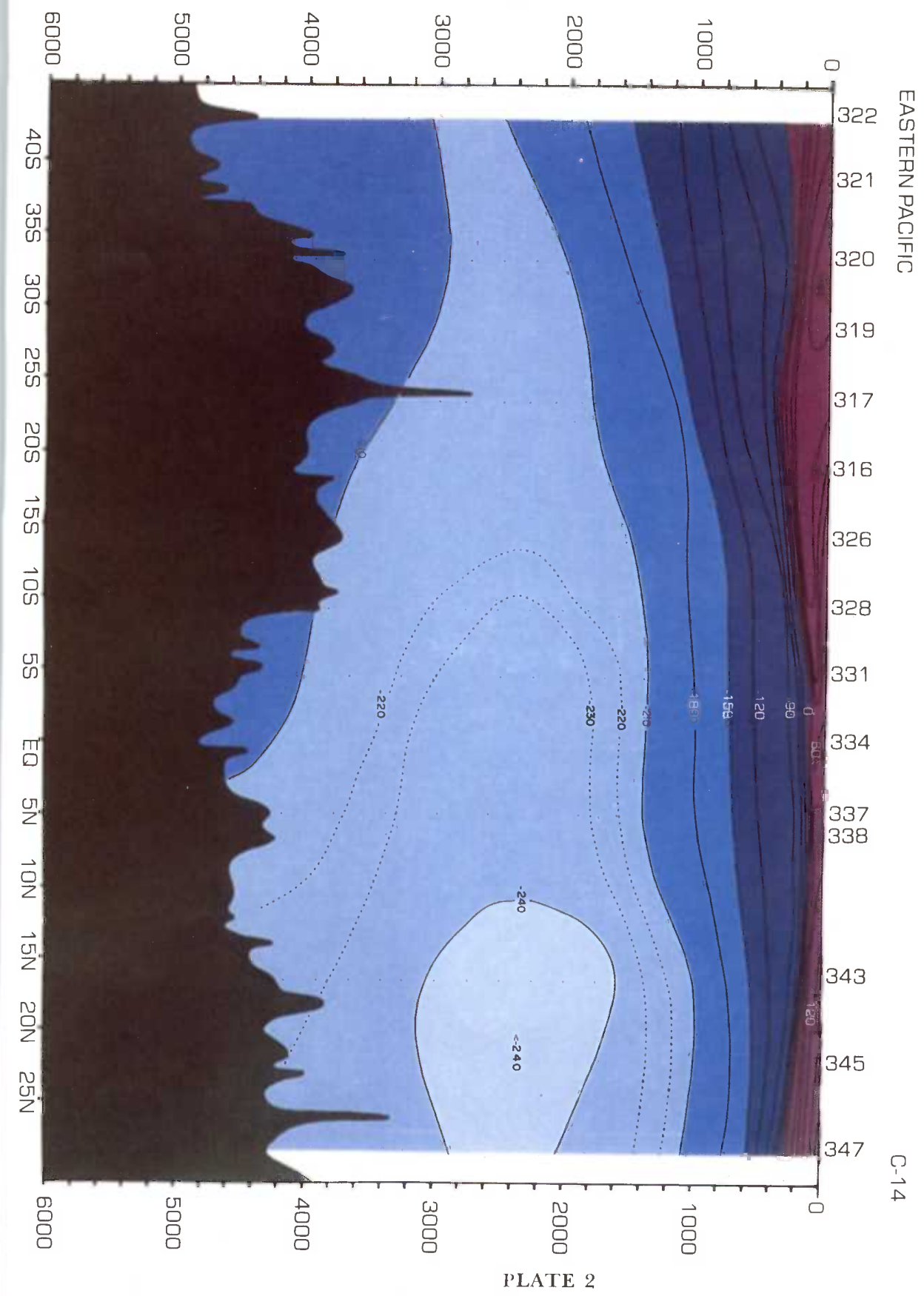

Eastern Pacific Ocean; N-S scction, mainly along $125^{\circ} \mathrm{W}$. 
GEOSECS Pacific Radiocarbon

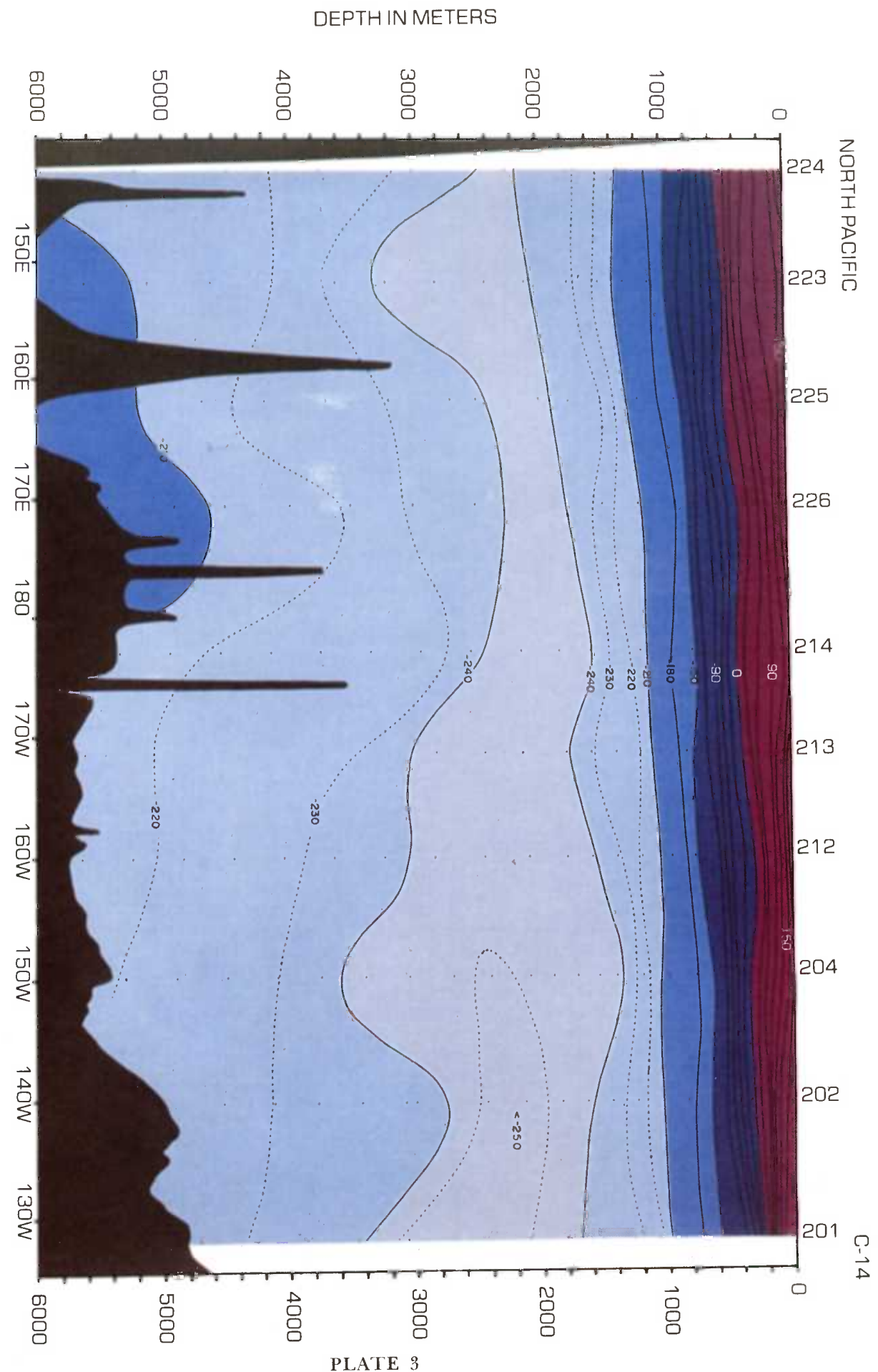

North Pacific Ocean; E-W section, mainly along $30^{\circ} \mathrm{N}$. 


\begin{tabular}{|c|c|c|c|c|c|c|c|}
\hline & & & & {$[0 N 201$} & & & \\
\hline & POSITI & 34 & $10 \mathrm{~N}$ & $12753 \mathrm{~W}$ & DATE & 25 AUG & 73 \\
\hline SMPL & DEPTH & POT T & SAL & SIGMA & $\mathrm{TCO} 2$ & DC 14 & C $14 \mathrm{LAB}$ \\
\hline 非 & M & DEG C & $0 / 00$ & THETA & UM & $0 / 00$ & \# \\
\hline 1187 & 2 & 17.60 & 33.085 & 23.933 & 2000 & 188.6 & ML 1305 \\
\hline 1189 & 67 & 14.75 & 33.080 & 24.582 & 1998 & 190.6 & ML 1304 \\
\hline 1190 & 141 & 10.09 & 33.208 & 25.581 & 2052 & 140.2 & ML 1303 \\
\hline 1191 & 216 & 8.48 & 33.860 & 26.349 & 2155 & 1.0 & ML 1302 \\
\hline 586 & 295 & 7.54 & 34.025 & 26.618 & 2217 & -75.4 & ML 1300 \\
\hline 587 & 394 & 6.40 & 34.062 & 26.802 & 2285 & -120.1 & ML 1298 \\
\hline 588 & 493 & 5.30 & 34.095 & 26.963 & 2314 & -138.0 & ML 1296 \\
\hline 589 & 592 & 4.91 & 34.195 & 27.088 & 2352 & -156.5 & ML 1299 \\
\hline 590 & 741 & 4.54 & 34.328 & & 2373 & -181.9 & ML 1294 \\
\hline 591 & 889 & 4.11 & 34.409 & 27.342 & 2395 & -198.4 & ML 1293 \\
\hline 592 & 1087 & 3.52 & 34.472 & 27.451 & 2387 & -225.0 & ML 1292 \\
\hline 593 & 1286 & 3.04 & 34.516 & 27.531 & 2395 & -234.6 & ML 1291 \\
\hline 594 & 1535 & 2.53 & .556 & 27. & 2405 & -235.7 & ML 1290 \\
\hline 286 & 1816 & 2.07 & 34.591 & 27.6 & 2404 & -244.5 & ML 1283 \\
\hline 287 & 2114 & 1.81 & 34.624 & 27.7 & 2398 & -250.2 & ML 1282 \\
\hline 390 & 3073 & 1.33 & 34.662 & & 2375 & -252.1 & ML 1288 \\
\hline 391 & 3471 & 1.22 & 34.674 & 27.800 & 2380 & -238.7 & ML 1287 \\
\hline 392 & 3868 & 1.16 & 34.681 & 27.8 & 2394 & -230.9 & ML 1286 \\
\hline & 4264 & 1.14 & & 27.812 & 2358 & -231.2 & ML 1285 \\
\hline 394 & 4707 & 1.13 & 34.684 & 27.814 & & -225.1 & ML 1284 \\
\hline
\end{tabular}

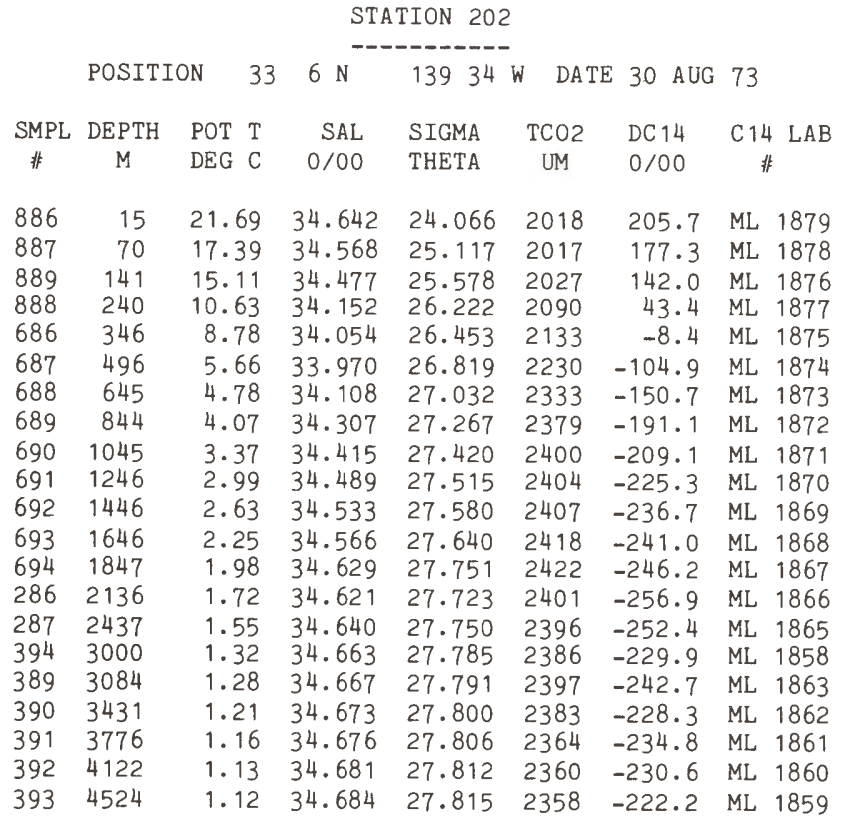




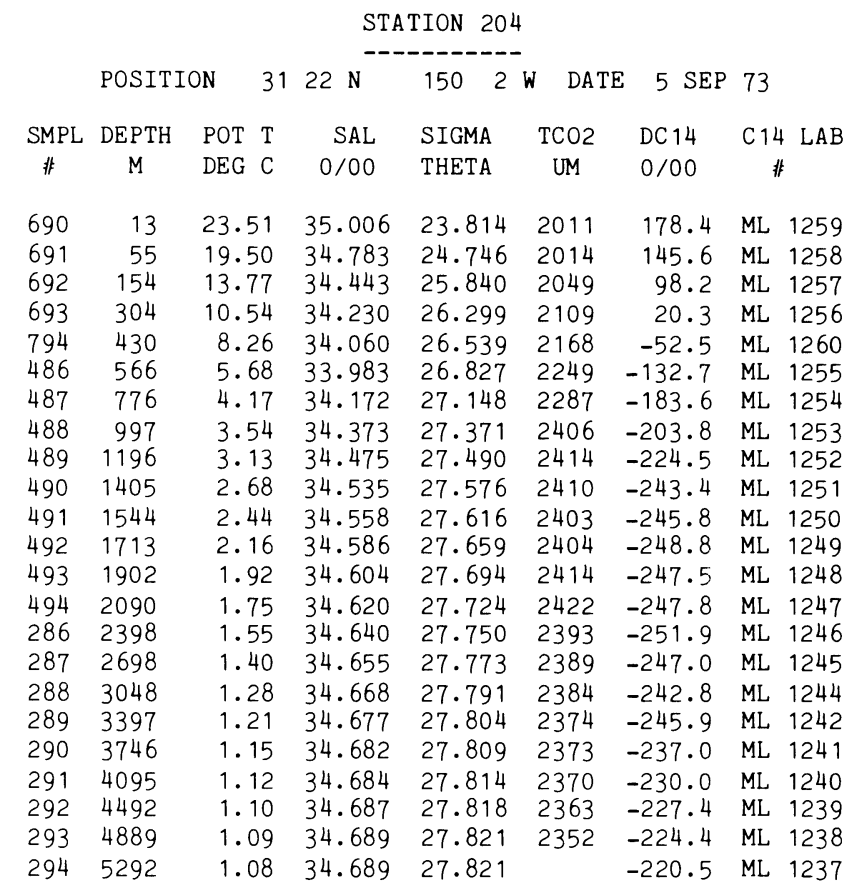

\begin{tabular}{|c|c|c|c|c|c|c|c|}
\hline & & & & ION 212 & & & \\
\hline & POSITI & 30 & $0 \mathrm{~N}$ & 15950 & DATE & $18 \mathrm{SEP}$ & 73 \\
\hline SMPL & DEPTH & POT $\mathrm{T}$ & SAL & SIGMA & TCO2 & DC 14 & C14 LAB \\
\hline \# & M & DEG C & $0 / 00$ & THETA & UM & $0 / 00$ & 非 \\
\hline 740 & 260 & 12.95 & 34.393 & 25.970 & 2054 & 4.1 & ML 1959 \\
\hline 486 & 426 & 9.36 & 34.141 & 26.428 & 2133 & -38.7 & ML 1958 \\
\hline 487 & 595 & 6.02 & 34.006 & 26.804 & 2230 & -124.8 & ML 1957 \\
\hline 488 & 771 & 4.27 & 34.158 & 27.127 & 2347 & -152.5 & ML 1956 \\
\hline 489 & 956 & 3.62 & 34.318 & 27.319 & 2379 & -222.9 & ML 1955 \\
\hline 590 & 1192 & 3.08 & 34.468 & 27.489 & 2384 & -216.8 & ML 1954 \\
\hline 591 & 1391 & 2.69 & 34.529 & 27.572 & 2394 & -232.2 & ML 1953 \\
\hline 592 & 1590 & 2.35 & 34.565 & 27.629 & 2383 & -240.5 & ML 1952 \\
\hline 593 & 1790 & 2.09 & 34.589 & 27.668 & 2376 & -244.6 & ML 1951 \\
\hline 594 & 2090 & 1.80 & 34.616 & 27.713 & 2407 & -243.2 & ML 1950 \\
\hline 286 & 2442 & 1.55 & 34.640 & 27.750 & 2379 & -241.4 & ML 1949 \\
\hline 287 & 2841 & 1.38 & 34.657 & 27.776 & 2370 & -246.2 & ML 1948 \\
\hline 288 & 3241 & 1.27 & 34.668 & 27.792 & 2368 & -233.6 & ML 1947 \\
\hline 289 & 3640 & 1.20 & 34.674 & 27.802 & 2353 & -242.6 & ML 1946 \\
\hline 290 & 4039 & 1.14 & 34.678 & 27.809 & 2344 & -223.6 & ML 1945 \\
\hline 291 & 4438 & 1.11 & 34.682 & 27.814 & 2349 & -226.0 & ML 1944 \\
\hline 292 & 4836 & 1.08 & 34.685 & 27.818 & 2338 & -223.2 & ML 1943 \\
\hline 293 & 5238 & 1.07 & 34.687 & 27.820 & 2335 & $-217 \cdot 5$ & ML 1942 \\
\hline 294 & 5638 & 1.06 & 34.687 & 27.822 & & -220.7 & ML 1941 \\
\hline
\end{tabular}




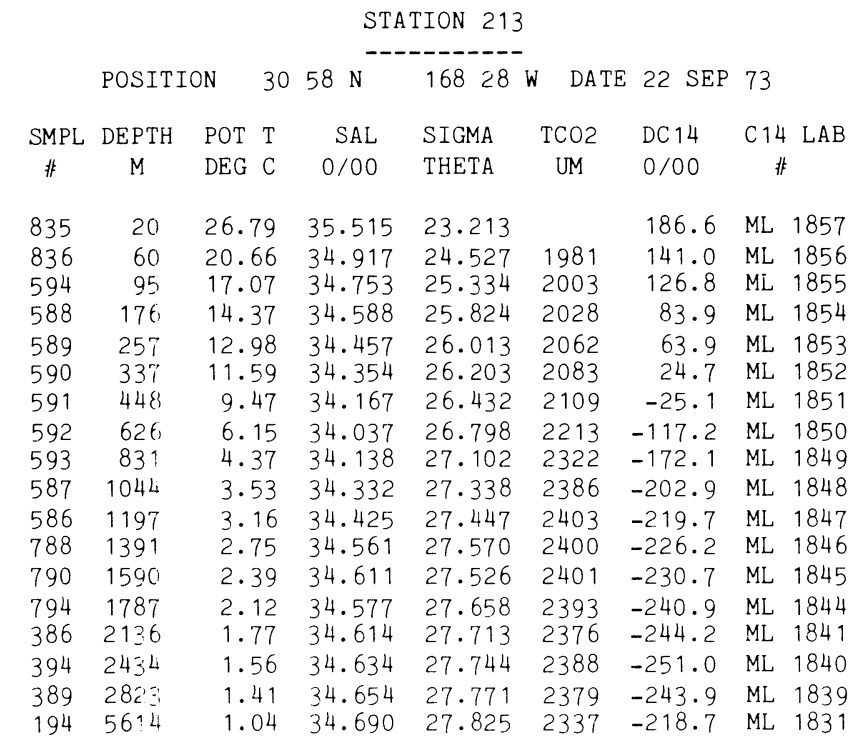

\begin{tabular}{|c|c|c|c|c|c|c|c|}
\hline & & & & ION 214 & & & \\
\hline & POSITI & 32 & $1 \mathrm{~N}$ & $17659 \mathrm{~h}$ & DATE & 25 SEP & 73 \\
\hline SMPL & DEPTH & POT $\mathrm{T}$ & SAL & SIGMA & $\mathrm{TCO} 2$ & DC 14 & C 14 LAB \\
\hline \# & M & DEG C & $0 / 00$ & THETA & UM & $0 / 00$ & 非 \\
\hline 935 & 20 & 25.66 & 34.751 & 22.983 & 1958 & 145.2 & ML 1236 \\
\hline 936 & 60 & 18.27 & 34.719 & 25.018 & 1980 & 105.4 & ML 1235 \\
\hline 886 & 105 & 15.65 & 34.664 & 25.601 & 2029 & 99.1 & ML 1234 \\
\hline 887 & 155 & 14.50 & 34.579 & 25.789 & 2036 & 84.7 & ML 1233 \\
\hline 889 & 276 & 12.41 & 34.414 & 26.094 & 2054 & 57.1 & ML 1231 \\
\hline 890 & 345 & 11.28 & 34.340 & 26.250 & 2082 & 40.2 & ML 1230 \\
\hline 891 & 474 & 8.43 & 34.131 & 26.568 & 2123 & -19.2 & ML 1228 \\
\hline 586 & 628 & 6.14 & 34.037 & 26.815 & 2227 & -102.7 & ML 1227 \\
\hline 587 & 777 & 4.59 & 34.117 & 27.060 & 2298 & -156.5 & ML 1226 \\
\hline 588 & 896 & 3.96 & 34.206 & 27.195 & 2357 & -174.1 & ML $\quad 1225$ \\
\hline 592 & 1191 & 3.08 & 34.400 & 27.435 & 2407 & -216.0 & ML 1224 \\
\hline 593 & 1591 & 2.32 & 34.531 & 27.605 & 2398 & -240.3 & ML 1223 \\
\hline 594 & 1840 & 2.00 & 34.574 & 27.664 & 2382 & -244.2 & ML 1222 \\
\hline 286 & 2143 & 1.71 & 34.610 & 27.715 & 2383 & -246.6 & ML 1220 \\
\hline 287 & 2542 & 1.48 & 34.640 & 27.755 & 2375 & -237.4 & ML 1219 \\
\hline 288 & 2942 & 1.32 & 34.658 & 27.781 & 2370 & -221.1 & ML, 1218 \\
\hline 289 & 3341 & 1.22 & 34.672 & 27.198 & 2371 & -221.3 & ML 1217 \\
\hline 290 & 3739 & 1.16 & 34.576 & 27.806 & 2376 & -228.1 & ML 1214 \\
\hline 291 & 4136 & i. 12 & 34.682 & 27.813 & 2359 & -220.4 & ML 1213 \\
\hline 292 & 4538 & 1.09 & 34.685 & 27.818 & 2345 & -222.6 & ML 1212 \\
\hline 293 & 4936 & 1.06 & 34.688 & 27.821 & 2350 & -211.8 & ML 1211 \\
\hline 294 & 5335 & & & & & -211.1 & ML 1210 \\
\hline
\end{tabular}


STATION 217

POSITION $4436 \mathrm{~N} 17650 \mathrm{~W}$ DATE 1 OCT 73

\begin{tabular}{|c|c|c|c|c|c|c|c|}
\hline SMPL & DEPTH & POT $\mathrm{T}$ & SAL & SIGMA & TCO2 & DC 14 & C $14 \mathrm{LAB}$ \\
\hline 非 & M & $\mathrm{DEG} C$ & $0 / 00$ & THETA & UM & $0 / 00$ & 非 \\
\hline 335 & 5 & 11.05 & 33.056 & 25.294 & & 79.2 & ML 1164 \\
\hline 336 & 5 & 11.05 & 33.056 & 25.294 & & 56.5 & ML 1163 \\
\hline 1088 & 95 & 6.43 & 33.609 & 26.440 & 2092 & 56.6 & ML 1172 \\
\hline 1089 & 155 & 6.80 & 33.769 & 26.516 & 2093 & 47.2 & ML $\quad 1174$ \\
\hline 1091 & 236 & 5.71 & 33.891 & 26.754 & 2183 & -43.7 & ML 1162 \\
\hline 1092 & 296 & 4.65 & 33.844 & 26.838 & 2238 & $-50 \cdot 3$ & ML 1167 \\
\hline 886 & 500 & 3.96 & 34.041 & 27.066 & 2326 & -145.2 & ML 1161 \\
\hline 887 & 645 & 3.67 & 34.181 & 27.204 & 2329 & -172.0 & ML 1160 \\
\hline 888 & 795 & 3.29 & 34.269 & $27 \cdot 312$ & 2332 & -193.9 & ML 1171 \\
\hline 889 & 994 & & 34.361 & 27.422 & 2365 & -204.4 & ML 1173 \\
\hline 890 & 1142 & & 34.410 & 27.480 & 2394 & $-214 \cdot 3$ & ML 1175 \\
\hline 891 & 1292 & 2.46 & 34.454 & 27.532 & 2407 & $-223 \cdot 5$ & ML 1156 \\
\hline 892 & 1490 & 2.19 & 34.507 & 27.595 & 2417 & -236.5 & ML 1176 \\
\hline 893 & 1788 & 1.94 & 34.560 & 27.657 & 2387 & -244.0 & ML 1168 \\
\hline 894 & 2085 & 1.76 & 34.591 & 27.696 & 2390 & -236.5 & ML 1155 \\
\hline 486 & 2556 & 1.51 & 34.632 & 27.746 & 2387 & -248.1 & ML 1158 \\
\hline 487 & 2952 & 1.37 & 34.653 & 27.774 & 2371 & -235.1 & ML 1157 \\
\hline 488 & 3348 & 1.26 & 34.666 & 27.791 & 2356 & $-222 \cdot 3$ & ML 1170 \\
\hline 489 & 3745 & 1.18 & 34.676 & 27.804 & 2350 & -226.8 & ML 1153 \\
\hline 490 & 4142 & 1.13 & 34.680 & 27.811 & 2344 & -224.7 & ML 1169 \\
\hline 491 & 4538 & 1.10 & 34.684 & 27.816 & 2354 & -218.0 & ML 1166 \\
\hline 492 & 4936 & 1.10 & 34.685 & 27.817 & 2354 & -218.4 & ML 1154 \\
\hline 493 & 5135 & 1.09 & 34.685 & 27.817 & 2363 & -217.6 & ML 1151 \\
\hline 494 & 5534 & & & & & -212.2 & ML 1159 \\
\hline
\end{tabular}

\begin{tabular}{|c|c|c|c|c|c|c|c|}
\hline & & & & ION 218 & & & \\
\hline & POSITI & 50 & $26 \mathrm{~N}$ & $17635 \mathrm{~W}$ & DATE & $4 \mathrm{OCT}$ & 73 \\
\hline SMPL & DEPTH & POT $\mathrm{T}$ & SAL & SIGMA & TCO2 & DC 14 & C $14 \mathrm{LAB}$ \\
\hline \# & M & DEG $C$ & $0 / 00$ & THETA & UM & $0 / 00$ & \# \\
\hline 241 & 10 & 8.25 & 32.649 & 25.436 & & 62.5 & ML 1209 \\
\hline 244 & 80 & 3.66 & 33.051 & 26.306 & 2112 & 52.8 & ML 1208 \\
\hline 594 & 144 & 3.52 & 33.415 & 26.609 & 2154 & -63.2 & ML 1207 \\
\hline 590 & 195 & 3.42 & 33.684 & 26.834 & 2242 & -77.4 & ML 1204 \\
\hline 586 & 344 & 3.49 & 33.968 & 27.053 & 2304 & -139.7 & Mi 1202 \\
\hline 587 & 543 & 3.35 & 34.162 & 27.221 & 2346 & -181.3 & ML 1201 \\
\hline 589 & 742 & 3.08 & 34.287 & 27.345 & 2347 & -191.0 & ML $\quad 1200$ \\
\hline 591 & 392 & 2.72 & 34.377 & 27.448 & 2396 & -216.4 & $\mathrm{~L} \quad 1139$ \\
\hline 592 & 1290 & 2.31 & 34.473 & 27.560 & 2398 & -223.4 & $M L \quad 1138$ \\
\hline $59 ?$ & 1589 & 2.03 & 34.529 & 27.627 & 2384 & -229.8 & ML 1191 \\
\hline 588 & 1888 & 1.83 & 34.573 & 27.575 & 2399 & -234.7 & ML, 1190 \\
\hline 892 & 2050 & 1.72 & 34.594 & 27.701 & 2401 & -240.2 & ML 1181 \\
\hline 394 & 2135 & 1.54 & 34.608 & 27.716 & 2397 & -234.6 & ML 1799 \\
\hline 390 & 2593 & 1.45 & 34.538 & 27.756 & 2374 & -240.9 & 1! 1188 \\
\hline 336 & 2992 & 1.33 & +4.656 & 27.778 & 2371 & $-232 \cdot 3$ & ML 1187 \\
\hline 387 & 3390 & 1.23 & 34.568 & 27.794 & 2350 & -236.3 & ML $\$ 196$ \\
\hline 399 & 3789 & 1.16 & 34.676 & $=7.995$ & 2330 & -222.3 & Hi 1195 \\
\hline 39 & 4,67 & 1.11 & $3+.68 \%$ & 27.613 & 2340 & -221.9 & HL $\quad 1984$ \\
\hline 332 & 40,95 & 199 & $34=684$ & 27.817 & 2337 & -214.5 & $M 2,189$ \\
\hline 393 & 4982 & 1.96 & 34.686 & 27.920 & 2332 & -212.7 & ML $\quad 182$ \\
\hline 388 & 5373 & 1.15 & 34.587 & 27822 & 2326 & -210.3 & 34,190 \\
\hline 889 & 6031 & $\therefore .04$ & 34.689 & 27.824 & 2309 & -210.3 & $14 \quad 119$ \\
\hline 891 & 8433 & $\therefore .04$ & 34.688 & 27.823 & 2326 & $-209 . ?$ & M. 173 \\
\hline 393 & 7233 & & & & & $-212 \cdot 3$ & He $11: 7$ \\
\hline
\end{tabular}




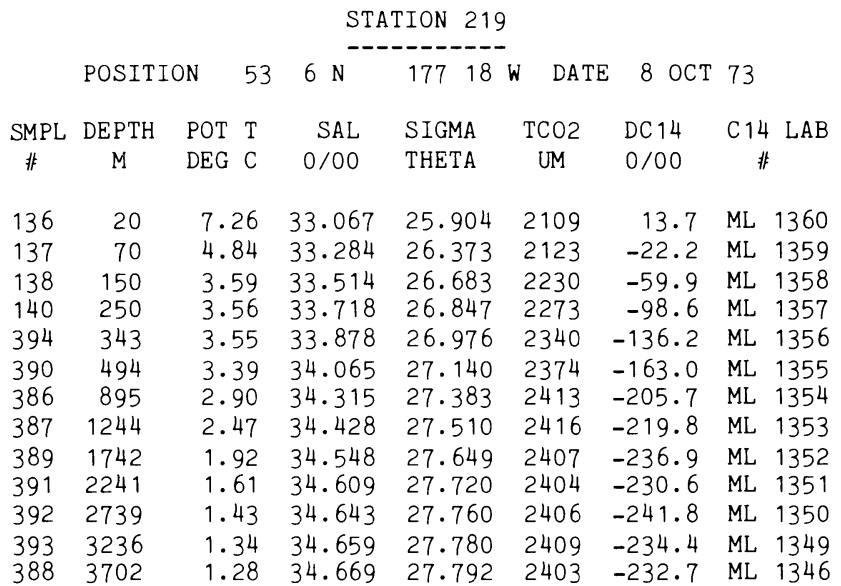

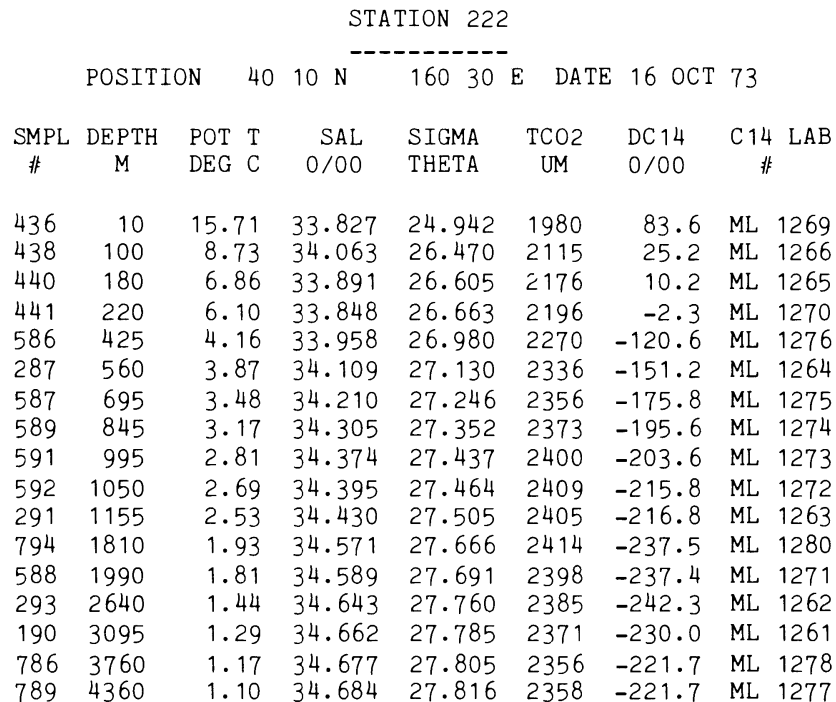


STATION 223

POSITION $3458 \mathrm{~N} \quad 15150 \mathrm{E}$ DATE 20 OCT 73

\begin{tabular}{|c|c|c|c|c|c|c|c|}
\hline SMPL & DEPTH & POT $\mathrm{T}$ & SAL & SIGMA & TCO2 & DC 14 & C14 LA \\
\hline \# & M & DEG $C$ & $0 / 00$ & THETA & UM & $0 / 00$ & 非 \\
\hline 335 & 10 & 22.47 & 34.524 & 23.747 & 1943 & 163.2 & ML \\
\hline & 70 & 20.5 & 34.777 & 24. & 199 & $140 \cdot 3$ & ML $18 \mathrm{C}$ \\
\hline & 125 & & & & 201 & & ML $18 \mathrm{C}$ \\
\hline & 200 & & & & 2016 & 122.9 & ML $\quad 18 c$ \\
\hline 39 & 300 & 16.62 & 34.742 & 25.435 & 2036 & 111.3 & ML 18 \\
\hline & 397 & & & & 2061 & & ML 1 \\
\hline 90 & 547 & & 34.431 & & 211 & & ML \\
\hline 86 & 697 & 7. & 34.210 & 26.695 & 2212 & -86.4 & ML 18 \\
\hline+87 & 897 & 4. & 34.109 & 69 & 2302 & 4 & ML \\
\hline 89 & 1097 & & 34.262 & & 23 & & ML \\
\hline+91 & 1296 & & 34.376 & 27. & 2391 & -204.3 & ML \\
\hline 93 & 95 & 2. & 34.446 & & 2409 & -2 & ML 17 \\
\hline 95 & 1744 & 2. & 34.515 & 27. & 2415 & $-232 \cdot 7$ & ML 17 \\
\hline 92 & 2091 & 1.9 & 34.575 & 27.671 & 2399 & -241.1 & ML 179 \\
\hline 19 & 2405 & & 34.615 & & 2402 & & ML \\
\hline & & & & & 2388 & & ML 17 \\
\hline 186 & 3205 & 1.33 & 34.660 & 27.782 & 2367 & -242.4 & ML 179 \\
\hline 187 & 3606 & & 34.671 & 27. & 2368 & & ML 17 \\
\hline & & & & & & & ML 17 \\
\hline 191 & 4610 & 1.10 & 34.684 & 27.816 & 2350 & -215.5 & ML \\
\hline & & & & 27.821 & 2344 & -211.1 & ML 17 \\
\hline & & & 34.691 & 27.826 & 2336 & -207.9 & ML 17 \\
\hline
\end{tabular}

STATION 224

POSITION $3415 \mathrm{~N} 14158$ E DATE 24 OCT 73

$\begin{array}{ccccccccc}\text { SMPL DEPTH } & \text { POT } & \text { T } & \text { SAL } & \text { SIGMA } & \text { TCO2 } & \text { DC14 } & \text { C14 } & \text { LAB } \\ \text { \# } & \text { M } & \text { DEG C } & 0 / 00 & \text { THETA } & \text { UM } & 0 / 00 & \text { \# } \\ & & & & & & & & \\ 435 & 20 & 23.84 & 34.503 & 23.339 & 1939 & 131.7 & \text { ML } 1912 \\ 436 & 80 & 21.58 & 34.703 & 24.125 & 1950 & 153.6 & \text { ML } 1918 \\ 437 & 150 & 18.65 & 34.774 & 24.962 & 1964 & 134.5 & \text { ML } 1917 \\ 438 & 250 & 17.07 & 34.763 & 25.343 & 1987 & 116.6 & \text { ML } 1916 \\ 391 & 400 & 15.69 & 34.692 & 25.612 & 2034 & 64.1 & \text { ML } 1914 \\ 389 & 505 & 13.57 & 34.539 & 25.956 & 2081 & 20.0 & \text { ML } 1915 \\ 393 & 800 & 6.73 & 34.193 & 26.861 & 2255 & -122.2 & \text { ML } 1913 \\ 294 & 997 & 4.29 & 34.208 & 27.156 & 2313 & -161.3 & \text { ML } 1911 \\ 290 & 1345 & 3.07 & 34.415 & 27.447 & 2374 & -210.6 & \text { ML } 1910 \\ 286 & 1745 & 2.23 & 34.519 & 27.602 & 2412 & -232.5 & \text { ML } 1909 \\ 287 & 2243 & 1.77 & 34.601 & 27.702 & 2399 & -241.8 & \text { ML } 1908 \\ 289 & 2988 & 1.39 & 34.654 & 27.772 & 2373 & -231.7 & \text { ML } 1907 \\ 291 & 3729 & 1.21 & 34.675 & 27.802 & 2348 & -225.0 & \text { ML } 1906 \\ 293 & 4471 & 1.11 & 34.685 & 27.816 & 2342 & -216.7 & \text { ML } 1905 \\ 295 & 5211 & 1.06 & 34.688 & 27.822 & 2348 & -214.6 & \text { ML } 1904 \\ 292 & 5950 & 1.04 & 34.694 & 27.827 & 2336 & -213.0 & \text { ML } 1903\end{array}$




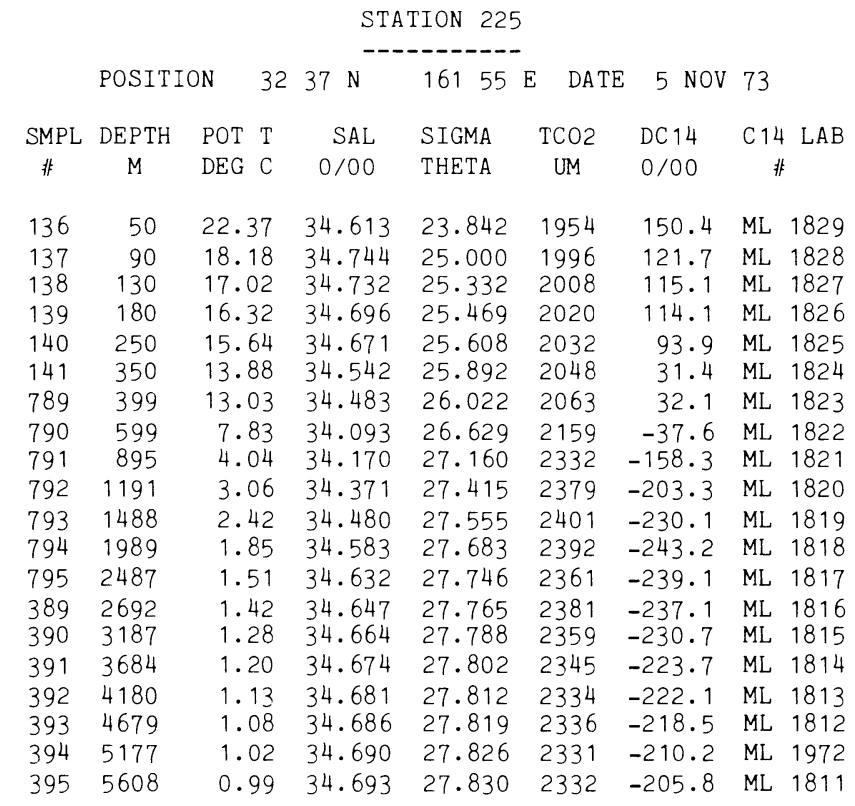

\begin{tabular}{|c|c|c|c|c|c|c|c|}
\hline & & & & ION 22 & & & \\
\hline & POSITI & $\mathrm{ON}$ & $34 \mathrm{~N}$ & $170 \quad 38$ & DATE & $9 \mathrm{NOV}$ & 73 \\
\hline SMPL & DEPTH & POT $\mathrm{T}$ & SAL & SIGMA & $\mathrm{TCO} 2$ & DC 14 & C $14 \mathrm{LAB}$ \\
\hline \# & M & DEG C & $0 / 00$ & THETA & UM & $0 / 00$ & 非 \\
\hline 135 & 10 & 24.73 & 35.055 & 23.494 & 1954 & 160.4 & ML 1321 \\
\hline 136 & 40 & 24.72 & 35.046 & 23.489 & 1965 & 168.3 & ML 1319 \\
\hline 137 & 80 & 23.65 & 35.006 & 23.774 & 1956 & 135.6 & ML 1318 \\
\hline 138 & 150 & 16.29 & 34.705 & 25.482 & 2005 & $100 \cdot 9$ & ML 1317 \\
\hline 139 & 250 & 14.47 & 34.572 & 25.790 & 2032 & 54.2 & ML 1316 \\
\hline 140 & 350 & 12.58 & 34.465 & 26.100 & 2058 & 11.9 & ML 1315 \\
\hline 389 & 452 & 10.43 & 34.283 & 26.359 & 2103 & -14.7 & ML 1323 \\
\hline 390 & 533 & 8.42 & 34.138 & 26.574 & 2134 & $-50 \cdot 3$ & ML 1325 \\
\hline 391 & 645 & 6.26 & 34.031 & 26.795 & 2208 & -95.7 & ML 1326 \\
\hline 392 & 904 & 3.91 & 34.194 & 27.192 & 2329 & -182.8 & ML 1327 \\
\hline 393 & 1192 & 3.04 & 34.406 & 27.443 & 2377 & -211.5 & ML 1328 \\
\hline 394 & 1480 & 2.47 & 34.501 & 27.569 & 2377 & -225.7 & ML 1329 \\
\hline 589 & 2499 & 1.46 & 34.641 & 27.758 & 2367 & -238.8 & ML 1337 \\
\hline 590 & 2999 & 1.29 & 34.662 & 27.786 & 2351 & -233.4 & ML 1336 \\
\hline 591 & 3495 & 1.18 & 34.675 & 27.803 & 2343 & -220.8 & ML 1334 \\
\hline 893 & 3980 & 1.12 & 34.682 & 27.813 & 2328 & -217.4 & ML 1338 \\
\hline 593 & 4499 & 1.06 & 34.687 & 27.821 & 2324 & -211.5 & ML 1333 \\
\hline 594 & 4901 & 1.01 & 34.691 & 27.827 & 2320 & $-207 \cdot 3$ & ML 1332 \\
\hline 595 & 5434 & 0.96 & 33.984 & 28.000 & 2311 & -203.7 & ML 1331 \\
\hline
\end{tabular}


STATION 227

POSITION $2459 \mathrm{~N} 170$ E DATE 12 NOV 73

\begin{tabular}{|c|c|c|c|c|c|c|c|}
\hline SMPL & DEPTH & POT $\mathrm{T}$ & SAL & SIGMA & TCO2 & DC 14 & $\mathrm{C} 14 \mathrm{LAE}$ \\
\hline 非 & M & DEG C & $0 / 00$ & THETA & UM & $0 / 00$ & 非 \\
\hline 135 & 10 & 26.60 & 35.212 & 23.042 & & 185.3 & ML \\
\hline 136 & 50 & 26.60 & 35.210 & 23.042 & 1952 & 173.4 & ML 13 \\
\hline 137 & 75 & 24.44 & 35.160 & 23.657 & 1965 & 161.0 & ML 13 \\
\hline 139 & 250 & 15.70 & 34.691 & 25.609 & 2010 & 69.3 & ML 13 \\
\hline 140 & 350 & & 34.465 & & & & ML \\
\hline 392 & 450 & 10.62 & 34.274 & 26.317 & 2073 & -21.6 & ML 13 \\
\hline 394 & 619 & 6.39 & 34.072 & 26.807 & 2228 & $-108 \cdot 5$ & ML 13 \\
\hline 387 & 897 & 3.92 & 34.280 & 27.259 & 2339 & -185.7 & ML 13 \\
\hline 389 & 1196 & 3.04 & 34.480 & 27.502 & 2361 & -218.0 & ML \\
\hline 390 & 1593 & 2.31 & 34.567 & 27.635 & 2374 & -229.7 & $M L$ \\
\hline 393 & 1991 & 1.85 & 34.613 & 27.706 & 2383 & -240.6 & ML 136 \\
\hline 395 & 2490 & 1.53 & 34.646 & 27.756 & 2378 & -240.2 & ML 136 \\
\hline 592 & 3137 & 1.31 & 34.666 & 27.788 & 2356 & -240.2 & ML 13 \\
\hline 94 & 3630 & 1.21 & 34.676 & 27.802 & 2341 & -230.5 & ML $13^{\circ}$ \\
\hline 587 & 4133 & 1.12 & 34.684 & 27.814 & 2333 & -214.0 & ML 137 \\
\hline 589 & 4630 & 1.04 & 34.690 & 27.825 & 2309 & -212.7 & ML $13^{\circ}$ \\
\hline 590 & 5128 & 0.98 & 34.694 & 27.831 & 2302 & -205.5 & ML 13 \\
\hline 593 & 5629 & 0.94 & 34.697 & 27.837 & 2318 & -199.6 & ML 13 \\
\hline 595 & 5957 & & & & & -196.9 & ML 136 \\
\hline
\end{tabular}

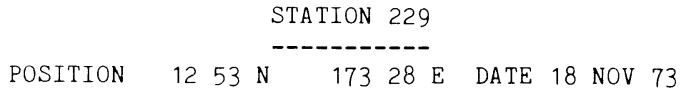

\begin{tabular}{|c|c|c|c|c|c|c|c|}
\hline SMPL & DEPTH & POT $\mathrm{T}$ & SAL & SIGMA & $\mathrm{TCO} 2$ & DC 14 & C 14 LAB \\
\hline \# & M & DEG C & $0 / 00$ & THETA & UM & $0 / 00$ & 非 \\
\hline 135 & 10 & 27.98 & 34.432 & 22.025 & & 132.8 & ML 1940 \\
\hline 136 & 50 & 27.94 & 34.437 & 22.040 & 1918 & 130.8 & ML 1939 \\
\hline 289 & 70 & 27.95 & 34.523 & 22.097 & 1927 & 129.2 & ML 1938 \\
\hline 290 & 96 & 27.00 & 34.849 & 22.653 & 1954 & 142.3 & ML 1937 \\
\hline 291 & 172 & 20.76 & 34.916 & 24.550 & 2018 & 152.8 & ML 1936 \\
\hline 293 & 249 & $13 \cdot 32$ & 34.416 & 25.911 & 2124 & 26.0 & ML 1935 \\
\hline 295 & 345 & 9.12 & 34.331 & 26.619 & 2233 & -87.9 & ML 1934 \\
\hline 492 & 444 & 7.14 & 34.336 & 26.918 & 2291 & -133.8 & ML 1933 \\
\hline 486 & 693 & 5.43 & 34.489 & 27.259 & 2328 & -163.4 & ML \\
\hline 489 & 993 & 4.22 & 34.544 & 27.438 & 2342 & -200.8 & ML 1931 \\
\hline 490 & 1291 & 3.29 & 34.578 & 27.557 & 2357 & -219.0 & ML 1930 \\
\hline 491 & 1590 & 2.50 & 34.606 & 27.649 & 2373 & -230.6 & ML 1929 \\
\hline 493 & 1888 & 2.17 & 34.624 & 27.691 & 2358 & -242.2 & ML 1927 \\
\hline 495 & 2286 & 1.77 & 34.647 & 27.740 & 2367 & -240.9 & ML 1926 \\
\hline 692 & 2684 & 1.55 & 34.659 & 27.765 & 2348 & -232.2 & ML 1925 \\
\hline 687 & 3184 & 1.34 & 34.672 & 27.790 & 2358 & -229.3 & ML 1924 \\
\hline 689 & 3684 & 1.19 & 34.679 & 27.806 & 2352 & -225.9 & ML 1923 \\
\hline 690 & 4186 & 1.05 & 34.688 & 27.822 & 2287 & -214.6 & ML 1922 \\
\hline 691 & 4686 & 0.94 & 34.694 & 27.834 & 2319 & -197.8 & ML 1921 \\
\hline 693 & 5188 & 0.87 & 34.700 & 27.843 & 2307 & -193.5 & ML 1920 \\
\hline 695 & 5689 & 0.86 & 34.700 & 27.844 & 2314 & -183.2 & ML 1919 \\
\hline
\end{tabular}


POSITION $146 \mathrm{~N} 17838 \mathrm{~W}$ DATE $21 \mathrm{NOV} 73$

$\begin{array}{ccccccccc}\text { SMPL } & \text { DEPTH } & \text { POT } & \text { T } & \text { SAL } & \text { SIGMA } & \text { TCO2 } & \text { DC 14 } & \text { C14 LAB } \\ \text { \#⿰ } & M & \text { DEG } & \text { C } & 0 / 00 & \text { THETA } & \text { UM } & 0 / 00 & \text { 非 }\end{array}$

$\begin{array}{lllllllll}135 & 10 & 27.22 & 34.634 & 22.416 & 1923 & 145.1 & \text { ML } & 1787\end{array}$

$\begin{array}{lllllllll}136 & 50 & 27.20 & 34.653 & 22.437 & 1938 & 144.9 & \text { ML } & 1786\end{array}$

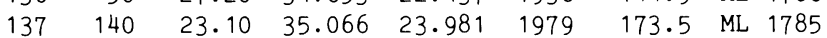

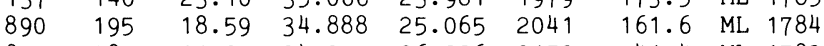

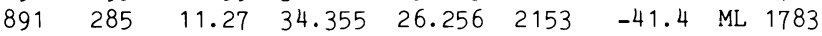

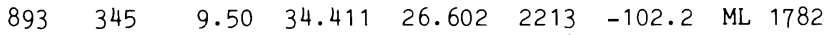

$\begin{array}{lllllllll}492 & 446 & 8.15 & 34.466 & 26.880 & 2258 & -121.3 & \text { ML } & 1779\end{array}$

$\begin{array}{lllllllll}487 & 796 & 5.31 & 34.515 & 27.296 & 2351 & -172.1 & \text { ML } & 1778\end{array}$

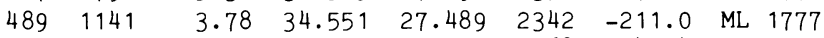

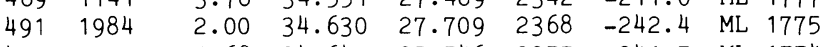

$\begin{array}{lllllllll}493 & 2379 & 1.68 & 34.647 & 27.746 & 2375 & -241.7 & \text { ML } & 1774\end{array}$

$\begin{array}{lllllllll}495 & 2574 & 1.58 & 34.655 & 27.760 & 2358 & -237.2 & \text { ML } & 1772\end{array}$

$\begin{array}{lllllllll}895 & 2775 & 1.47 & 34.662 & 27.773 & 2342 & -235.4 & \text { ML } & 1780\end{array}$

$\begin{array}{lllllllll}687 & 3597 & 1.19 & 34.678 & 27.805 & 2347 & -232.2 & \text { ML } & 1770\end{array}$

$\begin{array}{lllllllll}689 & 3995 & 1.10 & 34.684 & 27.816 & 2343 & -218.8 & \text { ML } & 1769\end{array}$

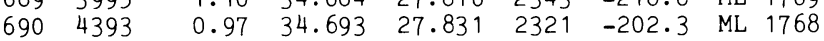

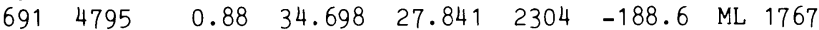

$\begin{array}{lllllllll}693 & 5194 & 0.85 & 34.700 & 27.845 & 2310 & -183.3 & \text { ML } & 1766\end{array}$

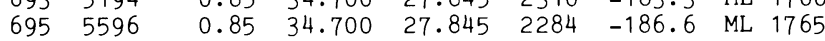

STATION 235

POSITION $1645 \mathrm{~N} 16119 \mathrm{~W}$ DATE 6 DEC 73

$\begin{array}{ccccrcccc}\text { SMPL DEPTH } & \text { POT } & \text { T } & \text { SAL } & \text { SIGMA } & \text { TCO2 } & \text { DC14 } & \text { C14 LAB } \\ \text { 非 } & M & \text { DEG } & \text { C } & 0 / 00 & \text { THETA } & \text { UM } & 0 / 00 & \text { 非 }\end{array}$

$\begin{array}{lllllllll}135 & 10 & 25.83 & 34.262 & 22.563 & 1928 & 144.7 & \text { ML } & 1902\end{array}$

$\begin{array}{lllllllll}136 & 40 & 25.85 & 34.383 & 22.631 & 1924 & 149.3 & \text { ML } & 1901\end{array}$

$\begin{array}{lllllllll}137 & 70 & 25.71 & 34.776 & 23.002 & 1964 & 151.0 & \text { ML } & 1900\end{array}$

$\begin{array}{lllllllll}138 & 130 & 21.76 & 34.905 & 24.238 & 1995 & 159.9 & \text { ML } & 1899\end{array}$

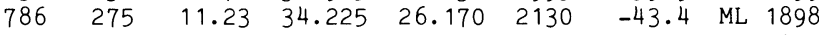

$\begin{array}{lllllllll}787 & 314 & 10.00 & 34.213 & 26.377 & 2174 & -81.8 & \text { ML } & 1897\end{array}$

$\begin{array}{lllllllll}793 & 354 & 8.81 & 34.191 & 26.555 & 2199 & -101.8 & \text { ML } & 1896\end{array}$

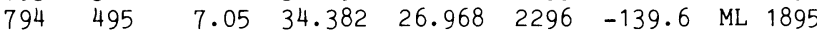

$\begin{array}{lllllllll}589 & 586 & 6.34 & 34.455 & 27.118 & 2313 & -144.6 & \text { ML } & 1893\end{array}$

$\begin{array}{lllllllll}795 & 592 & 6.29 & 34.457 & 27.125 & 2314 & -150.3 & \text { ML } & 1894\end{array}$

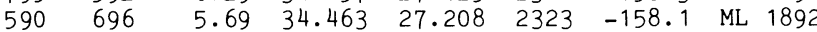

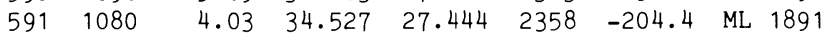

$\begin{array}{lllllllll}592 & 1496 & 2.81 & 34.584 & 27.606 & 2366 & -234.7 & \text { ML } & 1890\end{array}$

$\begin{array}{lllllllll}593 & 1894 & 2.14 & 34.618 & 27.689 & 2371 & -237.4 & \text { ML } & 1889\end{array}$

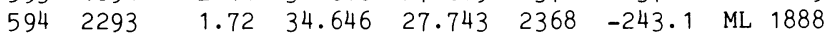

$\begin{array}{lllllllll}595 & 2690 & 1.50 & 34.660 & 27.769 & 2367 & -250.2 & \text { ML } & 1887\end{array}$

$\begin{array}{llllllll}387 & 3105 & 1.35 & 34.669 & 27.787 & 2363 & -246.0 & \text { ML } 1886\end{array}$

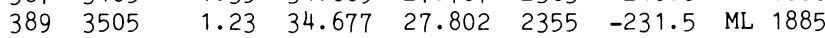

$\begin{array}{llllllll}390 & 3905 & 1.14 & 34.683 & 27.813 & 2350 & -221.2 & \text { ML } 1884\end{array}$

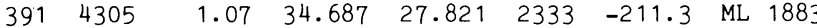

$\begin{array}{llllllll}392 & 4707 & 0.99 & 34.693 & 27.830 & 2322 & -207.8 & \text { ML } 1882\end{array}$

$\begin{array}{lllllllll}393 & 5108 & 0.95 & 34.695 & 27.835 & 2322 & -198.5 & \text { ML } & 1881\end{array}$

$\begin{array}{llllllll}394 & 5510 & 0.92 & 34.696 & 27.837 & 2308 & -200.3 & \text { ML } 1880\end{array}$ 


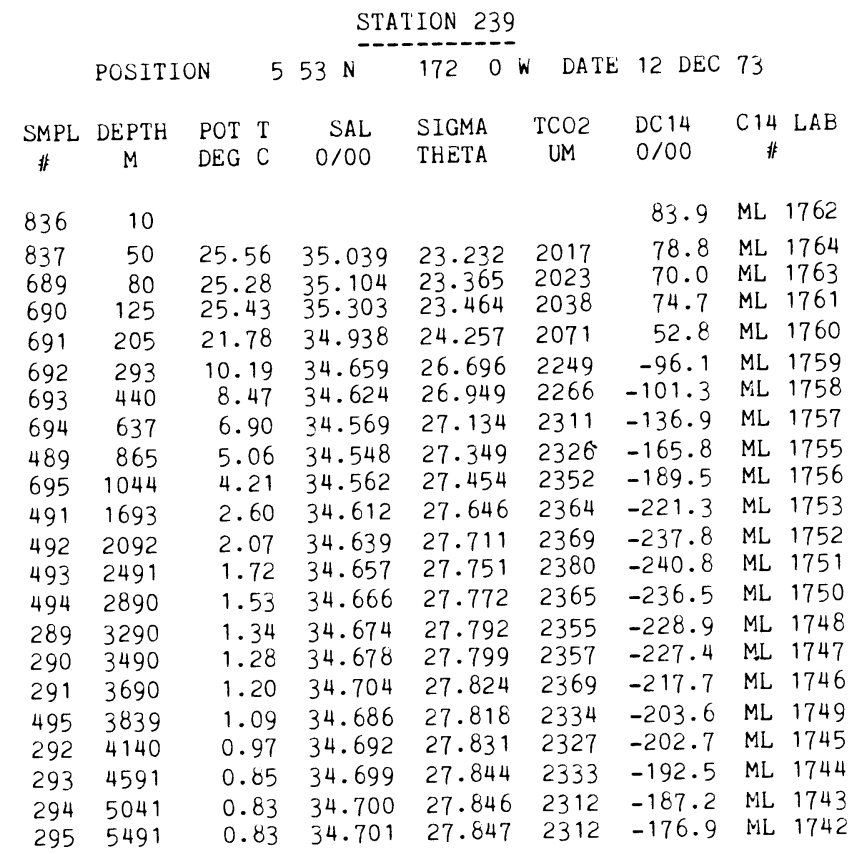

\begin{tabular}{|c|c|c|c|c|c|c|c|c|}
\hline & \multicolumn{8}{|c|}{ STATION 241} \\
\hline & POSITION & ON & $33 \mathrm{~N}^{---}$ & 1790 & DATE & $17 \mathrm{DEC}$ & 73 & \\
\hline SMPL & DEPTH & POT $\mathrm{T}$ & SAL & SIGMA & $\mathrm{TCO} 2$ & DC 14 & C 14 & $\mathrm{LAB}$ \\
\hline 非 & M & DEG C & $0 / 00$ & THETA & UM & $0 / 00$ & \# & \\
\hline 887 & 5 & 27.18 & 35.123 & 22.796 & 1996 & 87.4 & QL & 627 \\
\hline 889 & 50 & .15 & 35.131 & 22.809 & 2003 & 89.2 & QL & 628 \\
\hline 890 & 150 & 26.05 & 35.218 & 23.218 & 2038 & 81.8 & QL & 629 \\
\hline 891 & 230 & 16.08 & 34.696 & 25.520 & 2122 & 0.4 & QL & 630 \\
\hline 892 & 295 & 9.93 & 34.640 & 26.725 & 2252 & -102.7 & QL & 631 \\
\hline 893 & 425 & 8.31 & 34.610 & 26.961 & 2253 & -112.1 & QL & 632 \\
\hline 894 & 549 & 7.45 & 34.587 & 27.072 & 2282 & -121.1 & QL & 633 \\
\hline 895 & 674 & 6.50 & 34.563 & 27.183 & 2306 & $-134 \cdot 3$ & QL & 634 \\
\hline 886 & 947 & 4.92 & 34.542 & 27.362 & 2317 & -164.4 & QL & 635 \\
\hline 691 & 1102 & 4.28 & 34.554 & 27.442 & 2342 & -189.8 & QL & 636 \\
\hline 692 & 1501 & 2.94 & 34.599 & 27.606 & 2370 & -226.8 & QL & 637 \\
\hline 693 & 1898 & 2.20 & 34.631 & 27.693 & 2393 & -229.0 & QL & 638 \\
\hline 694 & 2247 & 1.80 & 34.651 & 27.740 & 2381 & $-234 \cdot 3$ & $\mathrm{QL}$ & 639 \\
\hline 686 & 2990 & 1.43 & 34.669 & 27.781 & 2380 & -227.7 & QL & 641 \\
\hline 286 & 3359 & 1.29 & 34.675 & 27.797 & 2360 & -222.9 & QL & 642 \\
\hline 287 & 3658 & 1.18 & 34.681 & 27.808 & 2349 & -216.5 & $\mathrm{QL}$ & 644 \\
\hline 289 & 3858 & 1.10 & 34.685 & 27.819 & 2351 & -216.8 & $\mathrm{QL}$ & 643 \\
\hline 290 & 4058 & 1.01 & 34.694 & 27.827 & 2336 & -202.8 & QL & 645 \\
\hline 291 & 4264 & 0.90 & 34.696 & 27.836 & 2330 & -194.6 & QL & 640 \\
\hline 292 & 4563 & 0.85 & 34.698 & 27.843 & 2334 & -181.0 & QL & 647 \\
\hline 293 & 4958 & 0.83 & 34.700 & 27.846 & 2308 & -187.2 & QL & 648 \\
\hline 294 & 5359 & 0.82 & 34.701 & 27.847 & 2307 & -187.5 & QL & 649 \\
\hline 295 & 5670 & 0.81 & 34.575 & 27.940 & 2321 & -185.0 & QL & 650 \\
\hline
\end{tabular}




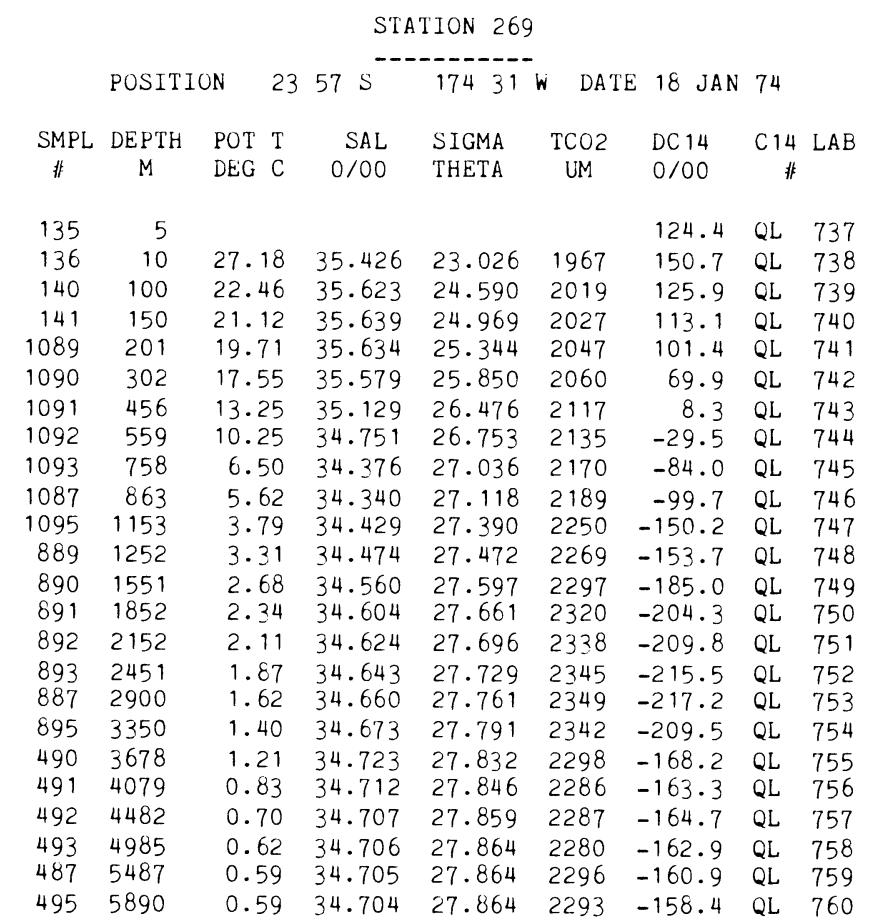

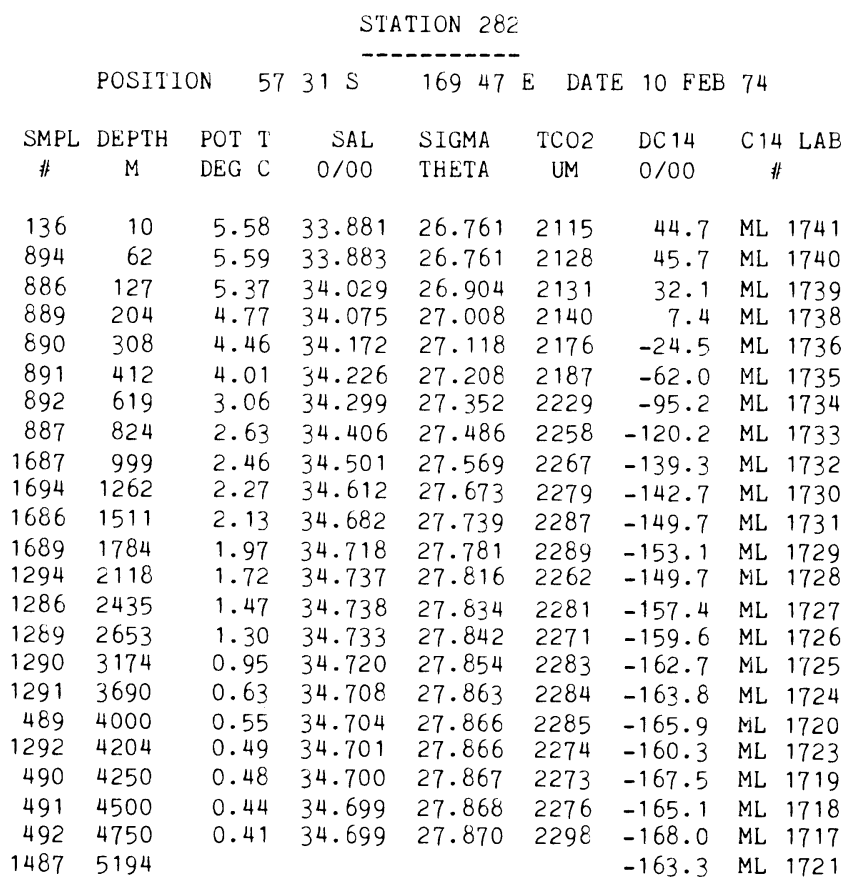




\begin{tabular}{|c|c|c|c|c|c|c|c|}
\hline & & & & ION 287 & & & \\
\hline & POSITI & 69 & $18 \mathrm{~s}$ & 17330 & DATE & 19 FEB & 74 \\
\hline SMPL & DEPTH & POT $\mathrm{T}$ & SAL & SIGMA & $\mathrm{TCO} 2$ & DC 14 & C14 LAB \\
\hline \# & M & DEG $C$ & $0 / 00$ & THETA & UM & $0 / 00$ & 非 \\
\hline 135 & 5 & -1.20 & 33.377 & 26.873 & 2137 & -76.4 & ML 1438 \\
\hline 894 & 66 & -1.61 & 34.277 & 27.613 & 2198 & -93.6 & ML 1437 \\
\hline 886 & 183 & 0.56 & 34.570 & 27.758 & 2246 & $-128 \cdot 3$ & ML 1436 \\
\hline 890 & 318 & 1.41 & 34.707 & 27.813 & 2256 & -152.9 & ML 1435 \\
\hline 891 & 537 & 1.29 & 34.725 & 27.837 & 2271 & -159.0 & ML 1434 \\
\hline 892 & 780 & 1.09 & 34.724 & 27.849 & 2277 & -156.1 & ML 1433 \\
\hline 889 & 1004 & 0.91 & 34.718 & 27.855 & 2279 & -161.6 & ML 1432 \\
\hline 694 & 1620 & 0.54 & 34.705 & 27.867 & 2282 & -162.6 & ML 1426 \\
\hline 686 & 1951 & 0.39 & 34.701 & 27.873 & 2282 & -161.1 & ML 1425 \\
\hline 690 & 2282 & 0.24 & 34.698 & 879 & 2283 & -162.5 & ML 1424 \\
\hline 691 & 2590 & 0.11 & 34.700 & 27.887 & 2284 & -163.5 & ML 1423 \\
\hline 692 & 2908 & 0.01 & 34.698 & 27.891 & 2284 & -159.9 & ML 1422 \\
\hline 689 & 3228 & -0.08 & 34.700 & 27.897 & 2273 & -155.5 & ML 1421 \\
\hline 1187 & 3233 & -0.09 & 34.700 & 27.897 & 2273 & -148.6 & ML 1431 \\
\hline $\begin{array}{l}487 \\
494\end{array}$ & $\begin{array}{l}3237 \\
3452\end{array}$ & $\begin{array}{l}-0.09 \\
-0.15\end{array}$ & $\begin{array}{l}34.700 \\
34.702\end{array}$ & & $\begin{array}{l}2273 \\
2274\end{array}$ & $\begin{array}{l}-159 \cdot 7 \\
-150 \cdot 9\end{array}$ & $\begin{array}{ll}\text { ML } & 1420 \\
\text { ML } & 1419\end{array}$ \\
\hline 1186 & 3460 & -0.15 & 34.702 & 27.901 & 2274 & -159.9 & ML 1430 \\
\hline 486 & 3614 & -0.18 & 34.768 & 27. & 2273 & -155.0 & ML 1418 \\
\hline 1190 & 3681 & -0.18 & 34.771 & 27.904 & 2273 & -154.2 & ML 1429 \\
\hline 490 & 3774 & -0.19 & 34.704 & 27.904 & 2274 & -144.6 & ML 1417 \\
\hline 1191 & 3902 & -0.21 & 34.705 & 27.905 & 2282 & -160.1 & ML 1428 \\
\hline 491 & 3941 & -0.21 & 34.703 & 27.905 & 2284 & $-153 \cdot 1$ & ML 1416 \\
\hline 492 & & & 34.705 & & 2274 & -151.0 & ML 1415 \\
\hline 1192 & 4093 & & 34.705 & & 2274 & -151.7 & ML 1427 \\
\hline
\end{tabular}

\begin{tabular}{|c|c|c|c|c|c|c|c|}
\hline & & & & ION 29 & & & \\
\hline & POSITI & 58 & $1 \mathrm{~S}$ & 1740 & DATE & $25 \mathrm{FEB}$ & 74 \\
\hline SMPL & DEPTH & POT $\mathrm{T}$ & SAL & SIGMA & $\mathrm{TCO} 2$ & DC 14 & C14 LAB \\
\hline 非 & M & DEG C & $0 / 00$ & THETA & UM & $0 / 00$ & 非 \\
\hline 836 & 10 & 4.53 & 33.958 & 26.941 & & 16.1 & ML 1716 \\
\hline 587 & 125 & 3.73 & 34.036 & 27.085 & 2157 & 8.8 & ML 1715 \\
\hline 594 & 200 & 3.24 & 34.065 & 27.154 & 2161 & -6.3 & ML 1713 \\
\hline 586 & 241 & 3.10 & 34.078 & 27.176 & 2166 & -14.4 & ML 1714 \\
\hline 590 & 321 & 3.28 & 34.184 & 27.244 & 2183 & -60.4 & ML 1712 \\
\hline 591 & 402 & 3.07 & 34.243 & 27.311 & 2212 & -85.6 & ML 1711 \\
\hline 592 & 500 & 2.85 & 34.308 & 27.381 & 2226 & -94.4 & ML 1710 \\
\hline 589 & 596 & 2.65 & 34.359 & 27.440 & 2238 & -114.1 & ML 1709 \\
\hline 387 & 704 & 2.49 & 34.417 & 27.500 & 2249 & $-131 \cdot 3$ & ML 1708 \\
\hline 394 & 905 & 2.34 & 34.530 & 27.602 & 2272 & -146.6 & ML 1707 \\
\hline 386 & 1203 & 2.20 & 34.647 & 27.707 & 2276 & -140.6 & ML 1706 \\
\hline 390 & 1499 & 2.04 & 34.704 & 27.765 & 2268 & -153.1 & ML 1705 \\
\hline 187 & 2307 & 1.42 & 34.736 & 27.836 & 2265 & -156.6 & ML 1703 \\
\hline 194 & 2704 & 1.10 & 34.726 & 27.850 & 2279 & $-163 \cdot 3$ & ML 1702 \\
\hline 186 & 3202 & 0.82 & 34.715 & 27.859 & 2276 & -161.7 & ML 1701 \\
\hline 190 & 3699 & 0.60 & 34.709 & 27.867 & 2282 & -160.1 & ML 1700 \\
\hline 191 & 4197 & 0.50 & 34.703 & 27.868 & 2294 & -169.3 & ML 1699 \\
\hline 192 & 4594 & 0.49 & 34.703 & 27.869 & 2291 & -161.6 & ML 1698 \\
\hline 189 & 4793 & 0.48 & 34.701 & 27.868 & 2289 & -164.5 & ML 1697 \\
\hline
\end{tabular}




\begin{tabular}{|c|c|c|c|c|c|c|c|}
\hline & \multicolumn{7}{|c|}{ STATION 293} \\
\hline & \multicolumn{2}{|c|}{ POSITION } & $35 \mathrm{~s}$ & 178 & DATE & $1 \mathrm{MAR}$ & 74 \\
\hline SMPL & DEPTH & POT $\mathrm{T}$ & SAL & SIGMA & $\mathrm{TCO} 2$ & DC 14 & C14 LAB \\
\hline 非 & M & DEG C & $0 / 00$ & THETA & UM & $0 / 00$ & 非 \\
\hline 1036 & 10 & 11.40 & 34.420 & 26.290 & 2057 & 56.2 & ML 1393 \\
\hline 1037 & 50 & 10.17 & 34.483 & 26.559 & 2078 & 56.9 & ML 1392 \\
\hline 587 & 101 & 8.49 & 34.642 & 26.825 & 2095 & 39. & ML 1391 \\
\hline 589 & 200 & 7.88 & 34.462 & 26.914 & 2106 & 26. & ML 1390 \\
\hline 590 & 302 & 7.20 & 34.411 & 26.969 & 2100 & 24.9 & ML 1389 \\
\hline 591 & 401 & 6.84 & 34.377 & 26.991 & 2107 & 19.3 & ML 1388 \\
\hline 592 & 501 & 6.30 & 34.334 & 27.029 & 2122 & -7.0 & ML 1387 \\
\hline 593 & 599 & 6.01 & 34.324 & 27.060 & 2137 & -33.0 & ML 1386 \\
\hline 594 & 695 & 5.22 & 34.270 & 27.108 & 2146 & -33.4 & ML 1385 \\
\hline 187 & 793 & 4.95 & 34.296 & 27.162 & 2148 & -58.6 & ML 1384 \\
\hline 194 & 992 & 3.93 & 34.316 & 27.289 & 2165 & -100.8 & ML 1383 \\
\hline 186 & 1191 & 3.11 & 34.359 & $27 \cdot 398$ & 2210 & -116.6 & ML 1382 \\
\hline 190 & 1589 & 2.45 & 34.525 & 27.589 & 2231 & -144.0 & ML 1611 \\
\hline 191 & 1987 & 2.21 & 34.651 & 27.709 & 2245 & $-164 \cdot 3$ & ML 1381 \\
\hline 192 & 2384 & 1.98 & 34.716 & 27.777 & 2247 & -154.1 & ML 1610 \\
\hline 189 & 2778 & 1.64 & 34.736 & 27.820 & 2260 & $-155 \cdot 3$ & ML 1380 \\
\hline 387 & 3230 & 1.29 & 34.731 & 27.842 & 2271 & -166.2 & ML 1609 \\
\hline 389 & 3726 & 0.92 & 34.719 & 27.855 & 2268 & -156.1 & ML 1379 \\
\hline 390 & & & 34.709 & & 2260 & -165.5 & ML 1608 \\
\hline 391 & 4620 & 0.54 & 34.702 & 27.865 & 2255 & $-161 \cdot 3$ & ML 1378 \\
\hline 392 & 4919 & 0.48 & 34.700 & 27.867 & 2255 & -164.4 & ML 1377 \\
\hline 393 & 5168 & 0.45 & 34.699 & 27.868 & 2262 & -168.4 & ML 1376 \\
\hline
\end{tabular}

\begin{tabular}{|c|c|c|c|c|c|c|c|}
\hline & \multicolumn{7}{|c|}{ STATION 296} \\
\hline & \multicolumn{2}{|c|}{ POSITION } & $56 \mathrm{~S}$ & 16639 & DATE & $16 \mathrm{MAR}$ & 74 \\
\hline SMPL & DEPTH & POT $\mathrm{T}$ & SAL & SIGMA & $\mathrm{TCO} 2$ & DC 14 & C $14 \mathrm{LAB}$ \\
\hline \# & M & DEG C & $0 / 00$ & THETA & UM & $0 / 00$ & \# \\
\hline 886 & 10 & 13.25 & 34.708 & 26.152 & & 79.5 & ML 1461 \\
\hline 889 & 99 & 10.95 & 34.900 & 26.729 & 2107 & 50.4 & ML 1459 \\
\hline 890 & 200 & 9.82 & 34.760 & 26.835 & 2111 & 47.6 & ML 1458 \\
\hline 892 & 400 & 8.36 & 34.571 & 26.924 & 2128 & 9.8 & ML 1457 \\
\hline 893 & 601 & 7.45 & 34.491 & 26.998 & 2149 & -56.9 & ML 1456 \\
\hline 895 & 803 & 6.44 & 34.418 & 27.077 & 2173 & -81.4 & ML 1455 \\
\hline 586 & 1002 & $5 \cdot 38$ & 34.398 & 27.194 & 2195 & -92.9 & ML 1454 \\
\hline 589 & 1252 & 4.17 & 34.416 & 27.340 & 2223 & -126.6 & ML 1453 \\
\hline 590 & 1503 & $3 \cdot 14$ & 34.465 & 27.485 & 2265 & -153.1 & ML 1452 \\
\hline 592 & 1754 & 2.72 & 34.561 & 27.595 & 2298 & -172.2 & ML 1451 \\
\hline 593 & 2004 & 2.34 & 34.609 & 27.664 & 2272 & -189.2 & ML 1450 \\
\hline 595 & 2254 & 2.13 & 34.644 & 27.710 & 2305 & $-192 \cdot 3$ & ML 1449 \\
\hline 386 & 2493 & 1.98 & 34.684 & 27.755 & 2287 & -175.0 & ML 1448 \\
\hline 389 & 2742 & 1.79 & 34.705 & 27.784 & 2283 & -156.6 & ML 1447 \\
\hline 390 & 2991 & 1.64 & 34.732 & 27.818 & 2264 & -160.7 & ML 1446 \\
\hline 392 & 3241 & 1.41 & 34.731 & 27.829 & 2268 & -160.0 & ML 1445 \\
\hline 394 & 3560 & 1.11 & 34.725 & 27.848 & 2276 & -166.0 & ML 1444 \\
\hline 187 & 3959 & 0.82 & 34.715 & 27.858 & 2273 & -166.4 & ML 1443 \\
\hline 189 & 4285 & 0.64 & 34.707 & 27.863 & 2292 & -166.5 & ML 1442 \\
\hline 191 & 4610 & 0.56 & 34.704 & 27.865 & 2274 & -169.0 & ML 1441 \\
\hline 193 & 4932 & 0.52 & 34.703 & 27.867 & 2271 & -167.5 & ML $\quad 1440$ \\
\hline 194 & 5252 & 0.50 & 34.523 & 27.803 & 2257 & -163.2 & ML $\quad 1439$ \\
\hline
\end{tabular}




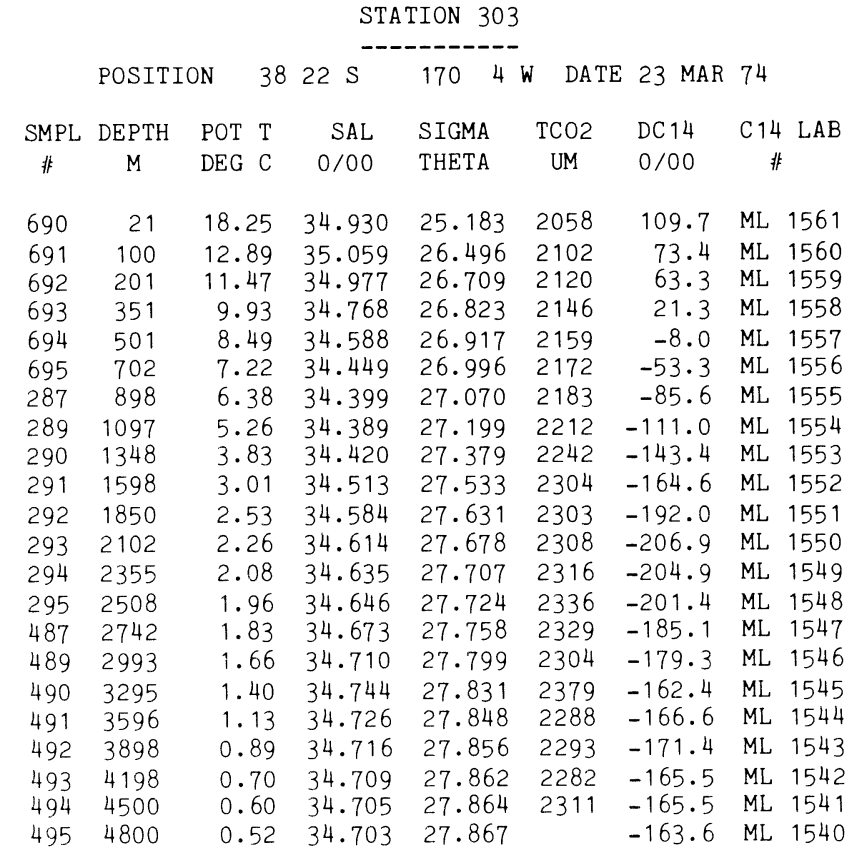

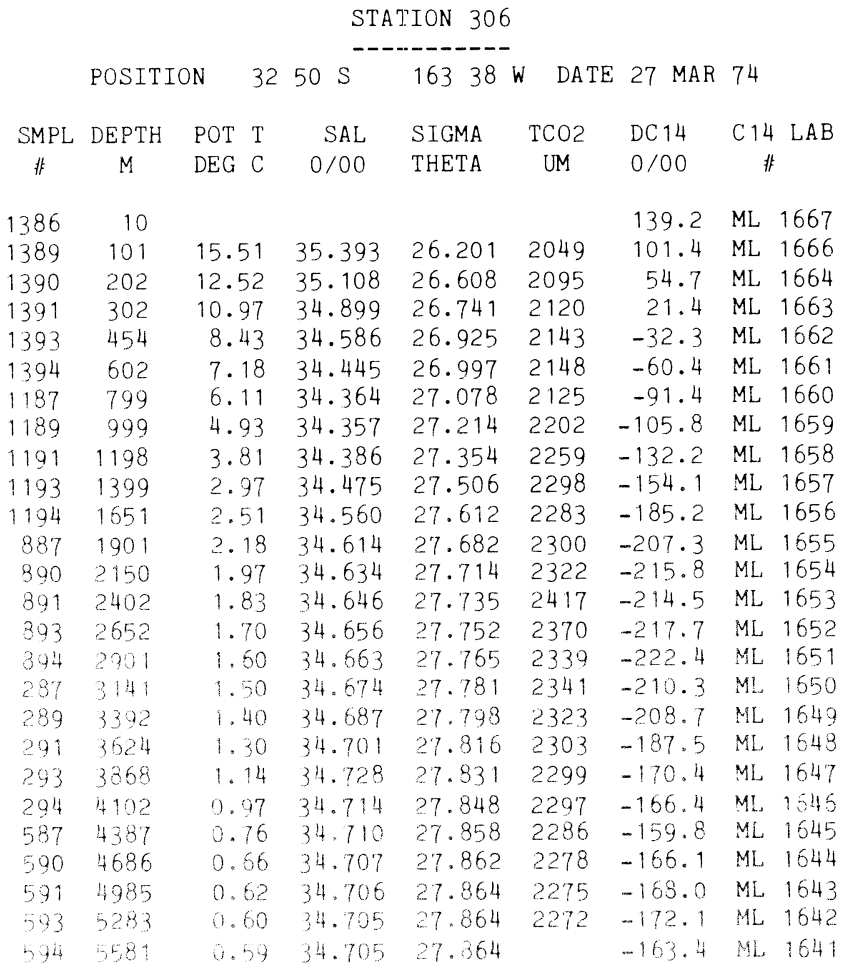




\begin{tabular}{|c|c|c|c|c|c|c|c|}
\hline \multirow[b]{3}{*}{ SMP } & \multicolumn{7}{|c|}{$\begin{array}{l}\text { STATION } 310 \\
-\end{array}$} \\
\hline & \multicolumn{2}{|c|}{ POSITION } & $57 \mathrm{~S}$ & 1579 & DATE & $3 \mathrm{APR}$ & 74 \\
\hline & DEPTH & POT T & SAL & SIGMA & TCO2 & DC 14 & C14 LAB \\
\hline 非 & M & DEG C & $0 / 00$ & THETA & UM & $0 / 00$ & \# \\
\hline 687 & 10 & & & & & $159 \cdot 3$ & ML 1692 \\
\hline 689 & 75 & 21.86 & 35.657 & 24.776 & 2024 & 137.0 & ML 1691 \\
\hline 690 & 135 & 19.68 & 35.598 & $25 \cdot 325$ & 2043 & 120.1 & ML 1690 \\
\hline 691 & 230 & 17.20 & 35.471 & 25.854 & 2071 & 88.9 & ML 1689 \\
\hline 692 & 330 & 14.45 & 35.198 & 26.276 & 2095 & 40.5 & ML 1688 \\
\hline 693 & 460 & 10.55 & 34.776 & 26.720 & 2126 & $-17 \cdot 5$ & ML 1687 \\
\hline 694 & 600 & 7.49 & 34.459 & 26.966 & 2148 & -56.8 & ML 1686 \\
\hline 695 & 750 & 6.20 & 34.358 & 27.061 & 2159 & -90.2 & ML 1685 \\
\hline 487 & 953 & 5.09 & 34.332 & 27.174 & 2187 & -101.6 & ML 1684 \\
\hline 489 & 1156 & 3.86 & 34.386 & $27 \cdot 350$ & 2228 & -139.4 & ML 1683 \\
\hline 490 & 1357 & 3.03 & 34.482 & 27.505 & 2267 & -164.8 & ML 1682 \\
\hline 491 & 1559 & 2.58 & 34.547 & 27.596 & 2293 & -175.9 & ML 1681 \\
\hline 492 & 1759 & 2.28 & 34.593 & 27.657 & 2316 & -200.7 & ML 1680 \\
\hline 493 & 1958 & 2.07 & 34.622 & 27.697 & 2346 & -208.1 & ML 1679 \\
\hline 494 & 2205 & 1.89 & 34.641 & 27.727 & 2334 & -217.8 & ML 1678 \\
\hline 495 & 2447 & 1.76 & 34.651 & 27.744 & 2354 & -219.7 & ML 1677 \\
\hline 286 & 2682 & 1.65 & 34.659 & 27.758 & 2341 & -221.2 & ML 1676 \\
\hline 287 & 2983 & 1.53 & 34.668 & 27.774 & 2332 & $-213 \cdot 1$ & ML 1675 \\
\hline 289 & 3281 & 1.43 & 34.677 & 27.789 & 2324 & -219.0 & ML 1674 \\
\hline 290 & 3580 & 1.25 & 34.687 & 27.814 & 2304 & $-200 \cdot 5$ & ML 1673 \\
\hline 291 & & 1.10 & 34.702 & 27.831 & 2293 & -180.2 & ML 1672 \\
\hline 292 & 4179 & 0.94 & 34.710 & 27.847 & 2293 & $-175 \cdot 3$ & ML 1671 \\
\hline 293 & 4577 & 0.75 & 34.710 & 27.859 & 2295 & -166.8 & ML 1670 \\
\hline 294 & 4976 & 0.68 & 34.710 & 27.863 & 2285 & -167.4 & ML 1669 \\
\hline 295 & 5379 & & & & & -165.6 & ML 1668 \\
\hline
\end{tabular}

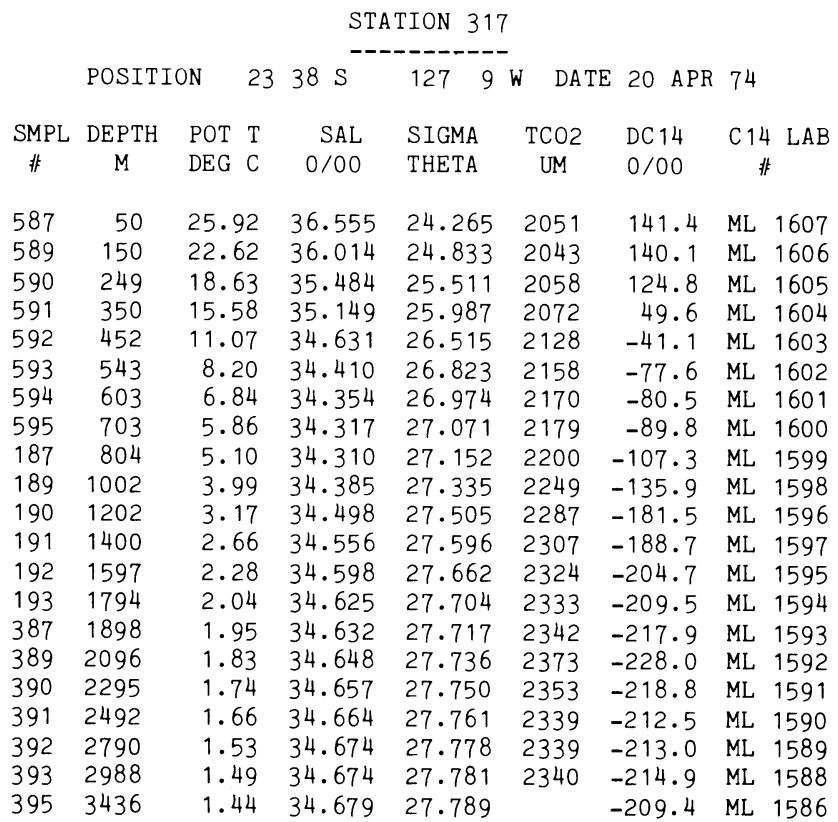


STATION 320

POSITION $3320 \mathrm{~S} 12824 \mathrm{~W}$ DATE $24 \mathrm{APR} 74$

\begin{tabular}{|c|c|c|c|c|c|c|c|}
\hline SMPL & DEPTH & POT $\mathrm{T}$ & SAL & SIGMA & TCO2 & DC 14 & C 14 LAB \\
\hline 非 & M & DEG C & $0 / 00$ & THETA & UM & $0 / 00$ & 非 \\
\hline 887 & 3 & 19.67 & 34.657 & 24.609 & 2040 & 171.1 & ML 1585 \\
\hline 889 & 59 & 19.05 & 34.795 & 24.869 & 2029 & 170.1 & ML 1584 \\
\hline 890 & 103 & $14 \cdot 70$ & 34.752 & 25.884 & 2032 & 151.9 & ML 1583 \\
\hline 891 & 163 & 12.92 & 34.649 & 26.173 & 2079 & 136.4 & ML 1582 \\
\hline 892 & 223 & 11.44 & 34.632 & 26.447 & 2105 & $77 \cdot 3$ & ML 1581 \\
\hline 893 & 303 & 9.42 & 34.542 & 26.731 & 2127 & -12.8 & ML 1580 \\
\hline 894 & 403 & 7.62 & 34.429 & 26.923 & 2180 & -54.8 & ML 1579 \\
\hline 895 & 504 & 6.81 & 34.382 & 27.000 & 2165 & $-58 \cdot 7$ & ML 1578 \\
\hline 187 & 606 & 6.34 & 34.351 & 27.038 & 2150 & -76.3 & ML 1577 \\
\hline 189 & 804 & 5.33 & 34.305 & $27 \cdot 126$ & 2178 & -87.9 & ML 1576 \\
\hline 190 & 1002 & 4.28 & 34.328 & 27.261 & 2213 & -115.2 & ML 1575 \\
\hline 191 & 1201 & $3 \cdot 37$ & 34.401 & 27.409 & 2261 & -143.7 & ML 1574 \\
\hline 192 & 1398 & 2.74 & 34.497 & 27.542 & 2297 & -162.1 & ML 1573 \\
\hline 193 & 1598 & 2.37 & 34.575 & 27.636 & 2304 & -182.8 & ML 1572 \\
\hline 194 & 1798 & 2.12 & 34.620 & 27.692 & 2309 & -192.2 & ML 1571 \\
\hline 195 & 2000 & 1.94 & 34.637 & 27.719 & 2324 & -208.7 & ML 1570 \\
\hline 487 & 2251 & 1.73 & 34.657 & 27.750 & 2323 & -217.2 & ML 1569 \\
\hline 489 & 2502 & 1.59 & 34.666 & 27.768 & 2326 & $-212 \cdot 7$ & ML 1568 \\
\hline 490 & 2753 & 1.49 & 34.674 & 27.781 & 2325 & -217.2 & ML 1567 \\
\hline 491 & 3002 & 1.41 & 34.679 & 27.791 & 2329 & -205.6 & ML 1566 \\
\hline 492 & 3252 & 1.35 & 34.683 & 27.799 & 2316 & $-212 \cdot 3$ & ML 1565 \\
\hline 493 & 3502 & 1.31 & & 27.804 & 2328 & -207.5 & ML 1564 \\
\hline 494 & 3949 & 1.20 & 34.693 & 27.816 & 2328 & $-199 \cdot 3$ & ML 1563 \\
\hline 495 & 4098 & 1.16 & 34.656 & 27.821 & 2312 & -195.5 & ML 1562 \\
\hline
\end{tabular}

STATION 322

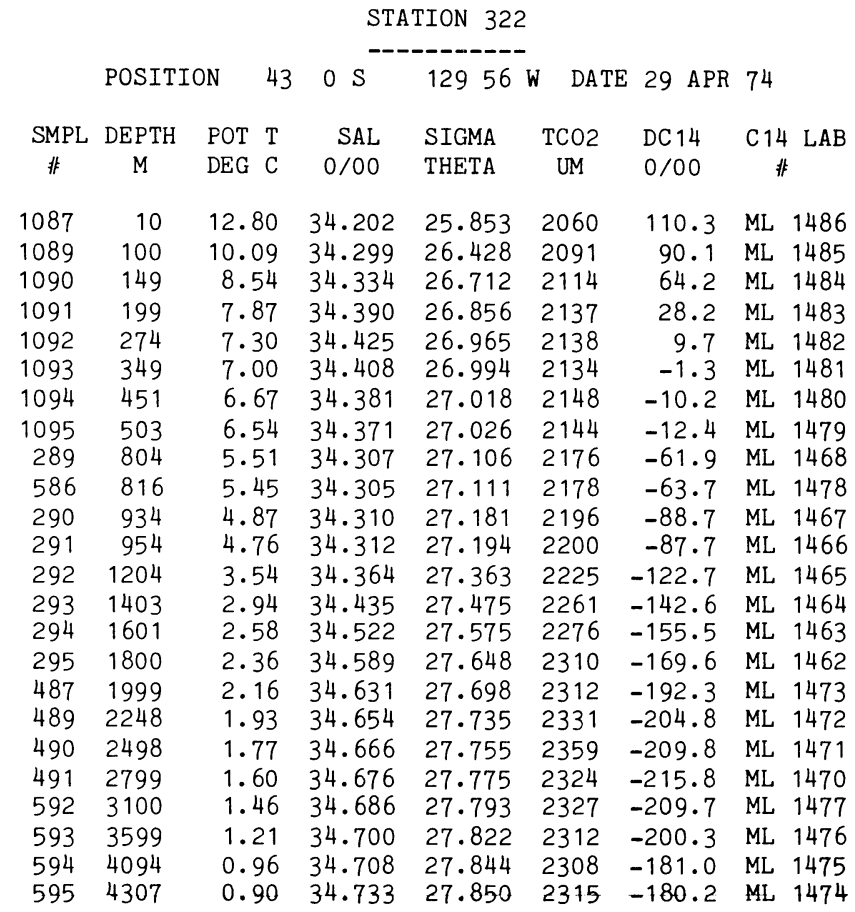




\begin{tabular}{|c|c|c|c|c|c|c|c|}
\hline & & & & ION 324 & & & \\
\hline & POSITI & & $0 \mathrm{~s}$ & 1464 & DATE & 7 MAY & 74 \\
\hline MPL & DEPTH & POT T & SAL & SIGMA & $\mathrm{TCO} 2$ & DC 14 & C14 LAB \\
\hline$\#$ & M & DEG $C$ & $0 / 00$ & THETA & UM & $0 / 00$ & $\#$ \\
\hline 36 & 10 & 26.57 & 35.942 & 23.601 & 1998 & 155.2 & ML 1630 \\
\hline & 100 & 23.33 & 3 & 24. & 201 & & ML 1629 \\
\hline & 150 & 20. & .682 & 25.0 & 202 & 132.7 & ML 1628 \\
\hline 90 & 240 & 18.27 & 35.506 & & 2050 & & ML 1627 \\
\hline 91 & 349 & 14.1 & .092 & & 208 & & ML 1626 \\
\hline 92 & 448 & 11. & & & 2129 & & ML 1625 \\
\hline 493 & 547 & 7.80 & 34.456 & 26. & 2159 & -70 & ML 1624 \\
\hline 94 & & & .342 & & 2170 & & ML 1623 \\
\hline 195 & 826 & 5.19 & 34.322 & 27. & 2201 & -107.6 & ML 1622 \\
\hline 186 & 1085 & 3.73 & 34.426 & 27 . & 2253 & -149.1 & ML 1621 \\
\hline 187 & & 2.3 & & & & & ML 1620 \\
\hline 189 & 208 & 1.93 & .638 & & 2338 & & ML 16 \\
\hline 190 & 2582 & 1.66 & 4.659 & & 2339 & -223 & ML 1617 \\
\hline 101 & 308 & 1.4 & & & & & ML 1616 \\
\hline 192 & & 1.22 & & & 2326 & -202.2 & ML 1615 \\
\hline 193 & 4082 & 1.09 & & & 2305 & & ML 1614 \\
\hline 194 & 458 & 1. & 34. & 27.831 & 2319 & & ML 161 \\
\hline 195 & 4982 & 1.03 & 34.699 & 27.833 & & -188.6 & ML 1612 \\
\hline
\end{tabular}

\begin{tabular}{|c|c|c|c|c|c|c|c|}
\hline & & & & TION 326 & & & \\
\hline & POSIT & & $3 s$ & $126 \quad 15$ & DATE & E $20 \mathrm{MAY}$ & 74 \\
\hline & DEPTH & POT T & SAL & SIGMA & $\mathrm{TCO} 2$ & DC 14 & C14 LAE \\
\hline 非 & M & DEG & $0 / 00$ & THETA & UM & $0 / 00$ & \\
\hline 36 & 10 & 25. & & & 2038 & 45 & ML 14 \\
\hline 37 & 110 & 24.35 & .423 & 24.662 & 2048 & 131.8 & ML 1495 \\
\hline 289 & 197 & 19.76 & 35.665 & 25.355 & 2085 & 139.1 & ML 1493 \\
\hline & 300 & 12.58 & & 26. & 2160 & -13.4 & ML 1494 \\
\hline & 399 & 8.39 & 34.573 & 26. & 2252 & -95.4 & ML 1492 \\
\hline 291 & 599 & 6.26 & 34.516 & 27.178 & 2250 & -118.0 & ML 1491 \\
\hline & 799 & & & & 2280 & -150.0 & ML 1490 \\
\hline & 1047 & 3. & 34.532 & 27. & 2300 & -176.2 & ML 1489 \\
\hline & 1295 & 3.23 & 34.564 & 27.553 & 2312 & -202.2 & ML 1488 \\
\hline & 1546 & 2.67 & 34.594 & 27.626 & 2319 & -212.2 & ML 1487 \\
\hline
\end{tabular}


STATION 328

\begin{tabular}{|c|c|c|c|c|c|c|c|c|}
\hline \\
\hline & POSIT & & 9 & $16 \mathrm{~S}$ & 12532 & DATE & $21 \mathrm{MAY}$ & 74 \\
\hline SMPL & DEPTH & POT & $\mathrm{T}$ & SAL & SIGMA & $\mathrm{TCO} 2$ & DC 14 & C14 LAB \\
\hline$\#$ & M & DEG & $\mathrm{C}$ & $0 / 00$ & THETA & UM & $0 / 00$ & \# \\
\hline 286 & 1830 & 2.3 & & 34.626 & 27.682 & 2332 & -216.9 & ML 1640 \\
\hline 287 & 2028 & 2.0 & & 34.640 & 27.712 & 2339 & -224.1 & ML 1639 \\
\hline 289 & 2277 & 1.8 & & 34.657 & 745 & 2340 & $-233 \cdot 2$ & ML 1638 \\
\hline 290 & 2527 & 1.0 & & 34.667 & 27.763 & 2344 & -230.4 & ML 1637 \\
\hline 291 & 2776 & 1.5 & & 34.673 & 27.774 & 2340 & -227.4 & ML 1636 \\
\hline 292 & 3026 & & & 34.679 & 27.788 & 2335 & $-221 \cdot 3$ & ML 1635 \\
\hline 293 & 3276 & 1.3 & & 34.687 & 27.803 & 2338 & -209.4 & ML 1634 \\
\hline 294 & 3527 & 1.2 & & 34.687 & 27.809 & 2337 & -211.4 & ML 1633 \\
\hline 295 & 3829 & 1.1 & & 34.691 & 27.817 & 2329 & -211.0 & ML 1631 \\
\hline
\end{tabular}

STATION 331

POSITION $438 \mathrm{~s}-1258 \mathrm{~W}$ DATE 23 MAY 74

\begin{tabular}{|c|c|c|c|c|c|c|c|}
\hline SMPL & DEPTH & POT $\mathrm{T}$ & SAL & SIGMA & $\mathrm{TCO} 2$ & DC 14 & C 14 LAB \\
\hline 非 & M & DEG C & $0 / 00$ & THETA & UM & $0 / 00$ & \# \\
\hline 236 & 10 & 26.43 & 35.137 & 23.040 & 2018 & 40.6 & ML \\
\hline 237 & 80 & & 35.460 & 24.996 & 2096 & 92.0 & ML \\
\hline 238 & 90 & 18.37 & 35.393 & 25.507 & 2116 & 65.0 & 1528 \\
\hline 239 & 100 & 16.59 & 35.284 & 25.857 & 2138 & 22.5 & ML 1527 \\
\hline 240 & 240 & 11 & 875 & 26.532 & 2250 & -77.2 & ML 1526 \\
\hline 489 & 302 & 11.03 & 34.820 & 26.668 & 2271 & -92.1 & ML 1525 \\
\hline 491 & 382 & 9.66 & 34.748 & 26.854 & 2262 & -102.6 & ML 1524 \\
\hline 492 & 503 & 8.00 & 34.630 & 27.025 & 2250 & $-108 \cdot 5$ & ML 1523 \\
\hline 494 & 632 & 6.69 & 34.574 & 27.165 & 2272 & $-130 \cdot 0$ & ML 1522 \\
\hline 886 & 798 & 5.20 & 34.540 & $27 \cdot 327$ & 2286 & -156.1 & ML 153 \\
\hline 887 & 1099 & 4.11 & 34.561 & 27.463 & 2319 & $-187 \cdot 3$ & ML 1538 \\
\hline 889 & 1400 & 3.21 & 34.588 & 27.573 & 2334 & -211.0 & ML \\
\hline 890 & 1701 & 2.52 & 34.617 & 27.657 & 2347 & -228.5 & ML \\
\hline 891 & 2002 & 11 & 34.639 & 27.707 & 2343 & -235.8 & ML 15 \\
\hline 892 & 2252 & 1.86 & 34.653 & 27.738 & 2353 & -231.6 & ML 1534 \\
\hline 893 & 2502 & 1.68 & 34.664 & 27.760 & 2355 & -241.7 & ML 1533 \\
\hline 894 & 2702 & 1.57 & 670 & 27.773 & 2350 & -235.2 & ML 1532 \\
\hline 295 & 3000 & 1.41 & 34.677 & 27.790 & 2345 & -229.9 & ML 1531 \\
\hline 190 & 3265 & 1.28 & 34.683 & 27.803 & 2337 & $-220 \cdot 3$ & ML 1521 \\
\hline 191 & 3657 & 1.12 & 34.690 & 27.819 & 2335 & $-213 \cdot 6$ & ML 1520 \\
\hline 193 & 4066 & 1.10 & 34.690 & 27.821 & 2327 & -209.8 & ML 1519 \\
\hline 195 & 4467 & & & & & -207.8 & ML \\
\hline
\end{tabular}




\begin{tabular}{|c|c|c|c|c|c|c|c|c|}
\hline \multicolumn{9}{|c|}{ STATION 334} \\
\hline & \multicolumn{2}{|c|}{ POSITION } & 0 & $3 \mathrm{~N}$ & $124 \quad 34$ & DATE & $27 \mathrm{MAY}$ & 74 \\
\hline SMPL & DEPTH & POT & $\mathrm{T}$ & SAL & SIGMA & $\mathrm{TCO} 2$ & DC 14 & C14 LAB \\
\hline \# & M & $\mathrm{DEG}$ & C & $0 / 00$ & THETA & UM & $0 / 00$ & 非 \\
\hline 236 & 10 & 24.8 & & 35.179 & 23.546 & 2039 & 72.6 & ML 1696 \\
\hline 237 & 110 & 19.0 & & 34.845 & 24.919 & 2092 & 47.5 & ML 1695 \\
\hline 238 & 160 & 14.0 & & 34.746 & 26.022 & 2141 & -19.5 & ML 1694 \\
\hline 239 & 195 & 12.9 & & 34.852 & 26.316 & 2155 & -45.6 & ML 1693 \\
\hline
\end{tabular}

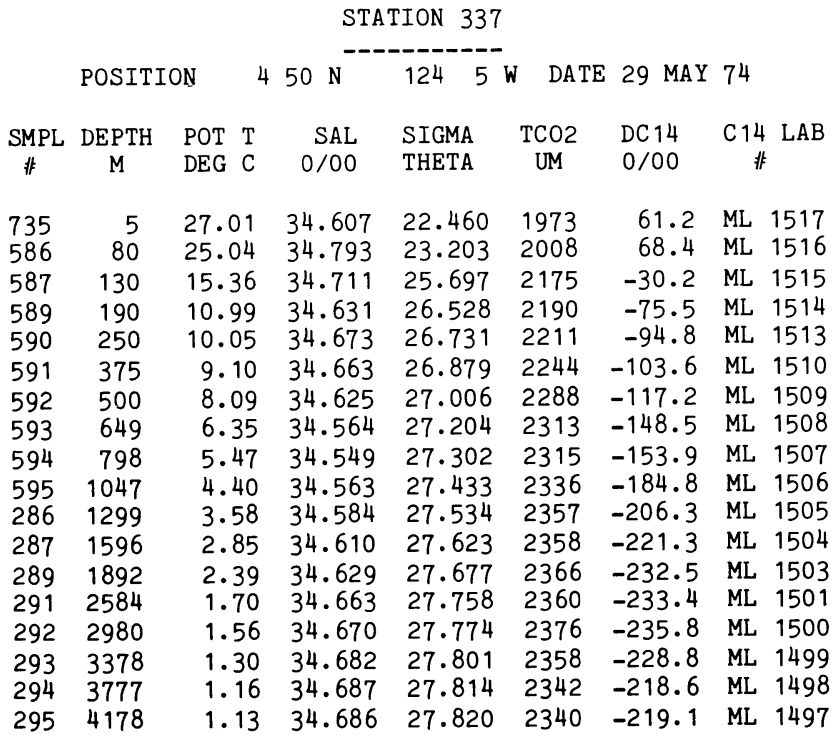


STATION 343

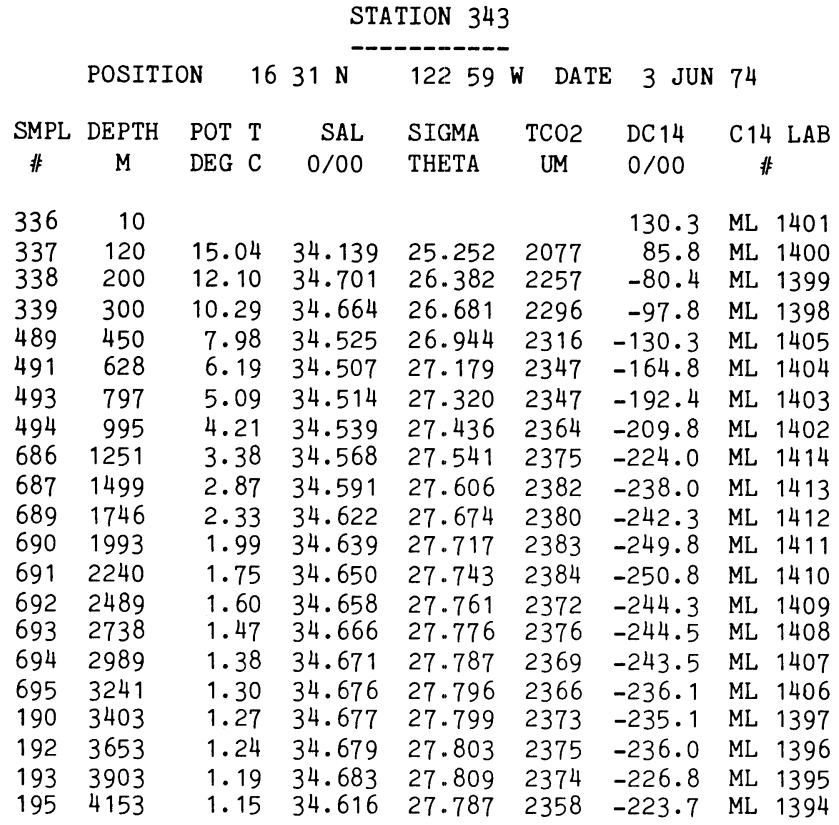

\begin{tabular}{|c|c|c|c|c|c|c|c|}
\hline & & & & ION 347 & & & \\
\hline & POSIT & ON & $30 \mathrm{~N}$ & 12129 & DATE & 8 JUN & 74 \\
\hline $1 \mathrm{PL}$ & DEPTH & POT $\mathrm{T}$ & SAL & SIGMA & TCO2 & DC 14 & C14 LAB \\
\hline 非 & M & DEG C & $0 / 00$ & THETA & UM & $0 / 00$ & \# \\
\hline 5 & 5 & 17. & & & & & ML 13 \\
\hline 86 & 599 & 5. & .403 & & 2340 & -15 & ML 13 \\
\hline 87 & 1501 & 2.80 & 34.573 & 27.597 & 2393 & -236.4 & ML 1312 \\
\hline 89 & 2003 & 1. & 31 & & 2385 & & ML 1311 \\
\hline 90 & 2304 & 1.7 & $34: 652$ & & 2376 & -247.2 & ML 1310 \\
\hline 32 & 2705 & 1.51 & 34.666 & 27.7 & 2370 & -244.6 & ML 1309 \\
\hline & & 1.4 & & & 2374 & & ML 1308 \\
\hline & 350 & 1.28 & 34.680 & 27. & 2363 & -241.0 & ML 1307 \\
\hline & 4001 & 1.22 & 34.684 & 27.8 & 2361 & -232.4 & ML 1306 \\
\hline
\end{tabular}

